\title{
ON THE INTERSECTION OF SETS OF INCOMING AND OUTGOING WAVES
}

\author{
BY \\ ADI DITKOWSKI (School of Mathematical Sciences, Tel Aviv University, Tel Aviv 69978, Israel) \\ AND
}

MICHAEL SEVER (Department of Mathematics, The Hebrew University of Jerusalem, Jerusalem, 91904, Israel)

\begin{abstract}
In the neighborhood of a boundary point, the solution of a first-order symmetric homogenous hyperbolic system is conveniently decomposed into fundamental waves solutions that are readily classified as outgoing, incoming, and stationary, or tangential.

Under a broad hypothesis, we show that the spans of the sets of outgoing and incoming waves have nontrivial intersection. Under these conditions, local, linear, perfectly nonreflecting local boundary conditions are shown to be an impossibility.
\end{abstract}

1. Introduction. Let us consider the symmetric hyperbolic system

$$
\tilde{\mathbf{u}}_{t}+\sum_{j=1}^{m} A_{j} \tilde{\mathbf{u}}_{\mathbf{x}_{j}}=\mathbf{g}(\mathbf{x}, t), \quad \mathbf{x} \in \mathbb{R}^{m}, \quad t>0, \quad \tilde{\mathbf{u}}(\mathbf{x}, t) \in \mathbb{R}^{n}, \quad m \geq 2,
$$

where

$$
A_{j} \in M_{n \times n} \quad \text { symmetric, } \quad j=1, \ldots, m
$$

and $\tilde{\mathbf{u}}(\mathbf{x}, 0)$ and $\mathbf{g}(\mathbf{x}, t)$ are such that

$$
\operatorname{supp}(\tilde{\mathbf{u}}(\mathbf{x}, 0)), \operatorname{supp}(\mathbf{g}(\mathbf{x}, t)) \subset \Omega_{0} \subset \mathbb{R}^{m}, \text { compact },
$$

where supp stands for support.

In many applications, it is desirable to locally decompose the general solution of equation (1.1) into "waves" that are moving in different directions. An example of such an application is the specification of nonreflecting, or absorbing, boundary conditions. Specifically, since in practice it is impractical to numerically solve problem (1.1), (1.3), over the whole $\mathbb{R}^{m}$, this problem is often approximated by

$$
\mathbf{u}_{t}+\sum_{j=1}^{m} A_{j} \mathbf{u}_{x_{j}}=\mathbf{g}(\mathbf{x}, t), \quad \mathbf{x} \in \Omega \subset \mathbb{R}^{m}, \quad t>0, \quad \mathbf{u}(\mathbf{x}, t) \in \mathbb{R}^{n}, \quad m \geq 2
$$

Received February 17, 2006.

2000 Mathematics Subject Classification. Primary 35Lxx, 35Qxx, 78Axx.

This research was supported by the Israel Science Foundation (grant No. 1364/04). 
for some bounded $\Omega \supseteq \Omega_{0}$ with the initial and boundary conditions

$$
\mathbf{u}(\mathbf{x}, 0)=\tilde{\mathbf{u}}(\mathbf{x}, 0)
$$

and

$$
B(\mathbf{x})\left(\left.\mathbf{u}(\mathbf{x}, t)\right|_{\mathbf{x} \in \partial \Omega}\right)=0
$$

with some $B: \partial \Omega \rightarrow M_{n \times n}(\mathbb{R})$. The objective is to choose $B$ independent of the solution $\tilde{\mathbf{u}}(\cdot, t), \mathbf{g}$, such that the solution $\mathbf{u}$ obtained from $(1.4),(1.5),(1.6)$ is unique and coincides with (or closely approximates) the restriction to $\Omega$ of the solution $\tilde{\mathbf{u}}$ as obtained from (1.1), (1.3). For more information about absorbing boundary conditions, see [1] and [2].

An application of the main result of this paper is to the possible form of such a boundary operator B. It is widely expected, at least for some specific examples of (1.1), that no such local operator $B$ exists. Below we shall show that this is indeed the case quite generally.

In order to understand the way the operator $B$ could be constructed, we consider the properties of the solution to (1.1) near the boundary $\partial \Omega$. Since $\mathbf{g}(\mathbf{x}, t)=\mathbf{0}$ near the boundary, we examine in this paper the homogenous system

$$
\mathbf{u}_{t}+\sum_{j=1}^{m} A_{j} \mathbf{u}_{x_{j}}=\mathbf{0}, \quad \mathbf{x} \in \Omega \subset \mathbb{R}^{m}, \quad t>0, \quad \mathbf{u}(\mathbf{x}, t) \in \mathbb{R}^{n}, \quad m \geq 2
$$

with the boundary condition (1.6), near a point $\mathbf{x}_{0} \in \partial \Omega$.

Equation (1.7) admits planar "wave" solutions of the form

$$
\mathbf{u}(\mathbf{x}, t)=\mathbf{r} f(\mathbf{e} \cdot \mathbf{x}-\lambda t), \quad \mathbf{e} \in \mathbb{R}^{m} /\{\mathbf{0}\}, \quad \mathbf{r} \in \mathbb{R}^{n} /\{\mathbf{0}\}, \quad f: \mathbb{R} \rightarrow \mathbb{R}
$$

satisfying

$$
(\mathbf{e} \cdot \mathbf{A}) \mathbf{r}=\lambda \mathbf{r}, \quad \mathbf{A}=\left(A_{1}, \ldots, A_{m}\right)^{T} .
$$

Here and below, · is the real $\mathbb{R}^{m}$ inner product.

The solutions (1.8) are waves that are moving in the direction of the vector $\mathbf{e}$ if $\lambda>0$, opposite to $\mathbf{e}$ if $\lambda<0$, and are stationary if $\lambda=0$. Throughout this paper, we consider the solution near a point $\mathbf{x}_{0} \in \partial \Omega$ with an outward normal $\boldsymbol{\nu}$. We will call the wave (1.8) outgoing if $(\mathbf{e} \cdot \nu) \lambda>0$, incoming if $(\mathbf{e} \cdot \nu) \lambda<0$, and stationary or tangential if $(\mathbf{e} \cdot \nu) \lambda=0$. For the case $\boldsymbol{\nu}=\hat{\mathbf{x}}=(1,0, \ldots, 0)^{T}$, we identify outgoing and incoming waves as right-moving and left-moving, respectively, in subsequent examples, depending on whether $(\mathbf{e} \cdot \hat{\mathbf{x}}) \lambda$ is respectively positive or negative.

Throughout the paper, we discuss polynomial wave solutions. These solutions are unbounded; however, they should be regarded as the first terms in the local Taylor expansion of the solutions near $\mathbf{x}_{0}$, rather than global solutions.

In the context of absorbing boundary conditions, proposed boundary conditions are often appraised by their treatment of specific local solutions corresponding to incoming and outgoing waves. A simple example shows that the classification of sets of waves as incoming or outgoing is ambiguous. 
EXAMPLE 1.1. Let us consider the following equation ${ }^{1}$ :

$$
\mathbf{u}_{t}+\left(\begin{array}{ccc}
0 & 0 & 0 \\
0 & 0 & -1 \\
0 & -1 & 0
\end{array}\right) \mathbf{u}_{x}+\left(\begin{array}{ccc}
0 & 0 & -1 \\
0 & 0 & 0 \\
-1 & 0 & 0
\end{array}\right) \mathbf{u}_{y}=\mathbf{0}
$$

in the domain $\Omega=\{(x, y) \mid x<0\}$. The point $\mathbf{x}_{0}$ will be taken as $\mathbf{x}_{0}=\left(x_{0}, y_{0}\right)=(0,0)$, and the outward unit normal is $\boldsymbol{\nu}=(1,0)$.

REMARK. In this paper, for the theoretical parts, we use $x_{1}, \ldots, x_{m}$ as the space variables. In this and the rest of the examples in this manuscript, we use $x, y, z$ instead of $x_{1}, x_{2}, x_{3}$, respectively.

Every function $\mathbf{u}$ of the form

$$
\mathbf{u}=\left(\begin{array}{c}
-\sin (\theta) \\
-\cos (\theta) \\
1
\end{array}\right) f(\cos (\theta) x+\sin (\theta) y-t)
$$

is a wave solution of (1.10). In particular, by taking $f(\xi)=\xi, \theta_{1}=0, \theta_{2,3}=$ $\pm \pi / 6, \theta_{4,5}= \pm \pi / 3$, and the corresponding vectors $\mathbf{e}_{j}=\left(\cos \left(\theta_{j}\right), \sin \left(\theta_{j}\right)\right)^{T}$, the functions

$$
\begin{aligned}
& \mathbf{u}_{\mathbf{e}^{(1)}}=\left(\begin{array}{r}
0 \\
-1 \\
1
\end{array}\right)(x-t), \quad \mathbf{u}_{\mathbf{e}^{(4)}}=\left(\begin{array}{r}
-\sqrt{3} / 2 \\
-1 / 2 \\
1
\end{array}\right)\left(\frac{1}{2} x+\frac{\sqrt{3}}{2} y-t\right), \\
& \mathbf{u}_{\mathbf{e}^{(2)}}=\left(\begin{array}{r}
-1 / 2 \\
-\sqrt{3} / 2 \\
1
\end{array}\right)\left(\frac{\sqrt{3}}{2} x+\frac{1}{2} y-t\right), \quad \mathbf{u}_{\mathbf{e}^{(5)}}=\left(\begin{array}{r}
\sqrt{3} / 2 \\
-1 / 2 \\
1
\end{array}\right)\left(\frac{1}{2} x-\frac{\sqrt{3}}{2} y-t\right), \\
& \mathbf{u}_{\mathbf{e}^{(3)}}=\left(\begin{array}{r}
1 / 2 \\
-\sqrt{3} / 2 \\
1
\end{array}\right)\left(\frac{\sqrt{3}}{2} x-\frac{1}{2} y-t\right),
\end{aligned}
$$

are wave solutions moving in the direction $\mathbf{e}^{(j)}$. In particular, $\mathbf{u}_{\mathbf{e}^{(1)}} \ldots \mathbf{u}_{\mathbf{e}^{(5)}}$ are all rightmoving waves. However,

$$
\begin{aligned}
\left(\begin{array}{l}
0 \\
1 \\
1
\end{array}\right)(x+t)= & -\frac{3 \sqrt{3}}{-12+7 \sqrt{3}} \mathbf{u}_{\mathbf{e}^{(1)}}+\frac{9-3 \sqrt{3}}{-12+7 \sqrt{3}} \mathbf{u}_{\mathbf{e}^{(2)}}+\frac{9-3 \sqrt{3}}{-12+7 \sqrt{3}} \mathbf{u}_{\mathbf{e}^{(3)}} \\
& +\frac{-3+\sqrt{3}}{-12+7 \sqrt{3}} \mathbf{u}_{\mathbf{e}^{(4)}}+\frac{-3+\sqrt{3}}{-12+7 \sqrt{3}} \mathbf{u}_{\mathbf{e}^{(5)}}
\end{aligned}
$$

is a left-moving wave solution of (1.10) of the form (1.11) with $\theta=-\pi / 2$.

This example illustrates the fact that, in general, solutions cannot be classified into disjoint subspaces of right- or left-moving waves.

\footnotetext{
${ }^{1}$ This is a "generic" equation. This is the electromagnetic TE equation for $u_{1}=E_{x}, u_{2}=-E_{y}$, and $u_{3}=H_{z}$, the TM equation for $u_{1}=-H_{x}, u_{2}=H_{y}$, and $u_{3}=E_{z}$, and the acoustics equation if we take $u_{1}=v, u_{2}=u$, and $u_{3}=-\rho$.
} 
REMARK. It can be verified that if, instead of using $f(\xi)=\xi$ in (1.11) and (1.12), one would have used any other smooth function, then the linear terms in the Taylor expansion of $\sum_{j=1}^{5} a_{j} \mathbf{u}_{\mathbf{e}^{(j)}}$ (where the $a_{j}$ are the ones used in (1.13)) would be the same as the linear term in the Taylor expansion of the incoming wave

$$
\left(\begin{array}{l}
0 \\
1 \\
1
\end{array}\right) f(x+t)
$$

A more elaborate, physical example for this phenomenon is presented in Appendix A.

It should be noted that this is a multidimensional phenomenon. In one space dimension, $m=1$, the system (1.7) can be diagonalized, and it separates into a system of uncoupled advection equations.

In section 2, the phenomenon of coupling between incoming and outgoing waves is presented in detail.

In sections 3 and 4 , we show that this phenomenon is quite general. In particular, at a noncharacteristic boundary point which is neither "purely incoming" nor "purely outgoing", the spaces of incoming and outgoing waves necessarily having nontrivial intersection and perfectly nonreflecting boundary condition of the form (1.6) are impossible.

\section{Local wave expansion.}

2.1. Formulation. Formulation of boundary conditions for (1.7) depending on incoming and outgoing waves is based on a local wave expansion of solutions.

Let $\mathbf{u}(\mathbf{x}, t)$ be a solution of (1.7) of class $C^{1}$ near $\mathbf{x}_{0} \in \partial \Omega$ and $t_{0}$. Thus

$$
\mathbf{u}(\mathbf{x}, t)=\mathbf{u}\left(\mathbf{x}_{0}, t_{0}\right)+\left(t-t_{0}\right) \mathbf{u}_{t}\left(\mathbf{x}_{0}, t_{0}\right)+\sum_{j=1}^{m}\left(x_{j}-x_{0 j}\right) \mathbf{u}_{x_{j}}\left(\mathbf{x}_{0}, t_{0}\right)+o\left(\left|\mathbf{x}-\mathbf{x}_{0}\right|+\left|t-t_{0}\right|\right) .
$$

The linear part of $(2.1)$ is isomorphic to $\mathbb{R}^{m n}$ :

$$
\boldsymbol{\Phi}=\left(\begin{array}{c}
\mathbf{u}_{x_{1}}\left(\mathbf{x}_{0}, t_{0}\right) \\
\vdots \\
\mathbf{u}_{x_{m}}\left(\mathbf{x}_{0}, t_{0}\right)
\end{array}\right) \in \mathbb{R}^{m n}
$$

Since the time derivative is then obtained from (1.7),

$$
\mathbf{u}_{t}\left(\mathbf{x}_{0}, t_{0}\right)=-\sum_{j=1}^{m} A_{j} \mathbf{u}_{x_{j}}\left(\mathbf{x}_{0}, t_{0}\right)=-\left(A_{1}\left|A_{2}\right| \ldots \mid A_{m}\right) \mathbf{\Phi} .
$$

We can now choose $m n$ vectors $\mathbf{e}^{(\ell)}=\left(e_{1}^{(\ell)}, \ldots, e_{m}^{(\ell)}\right)^{T} \in \mathbb{R}^{m} /\{\mathbf{0}\}$, not necessarily different, indices $k_{\ell}$, and corresponding eigenvectors $\mathbf{r}_{k_{\ell}}$, satisfying

$$
\left(\mathbf{e}^{(\ell)} \cdot \mathbf{A}\right) \mathbf{r}_{k_{\ell}}\left(\mathbf{e}^{(\ell)}\right)=\lambda_{k_{\ell}} \mathbf{r}_{k_{\ell}}\left(\mathbf{e}^{(\ell)}\right) ; \quad k_{\ell} \in\{1, \ldots, n\}, \quad \ell=1, \ldots, m n .
$$

Note that for every vector $\mathbf{e}^{(\ell)}$ there is a choice of $n$ eigenvectors $\mathbf{r}_{k_{\ell}}$ and their corresponding eigenvalues $\lambda_{k_{\ell}}$. In order to simplify the notation we abbreviate $\mathbf{r}_{\ell}=\mathbf{r}_{k_{\ell}}$ and $\lambda_{\ell}=\lambda_{k_{\ell}}$, with the understanding that if $\mathbf{e}^{(\ell)}=\mathbf{e}^{\left(\ell^{\prime}\right)}$ for $\ell \neq \ell^{\prime}$, then they correspond to different eigenvalues/eigenvectors. Thus (2.4) becomes

$$
\left(\mathbf{e}^{(\ell)} \cdot \mathbf{A}\right) \mathbf{r}_{\ell}=\lambda_{\ell} \mathbf{r}_{\ell} ; \quad k_{\ell} \in\{1, \ldots, n\}, \quad \ell=1, \ldots, m n,
$$


and the $\mathbf{e}^{(\ell)}$ are chosen such that the $\boldsymbol{\Phi}_{\ell}$ given by

$$
\boldsymbol{\Phi}_{\ell}=\left(\begin{array}{c}
e_{1}^{(\ell)} \mathbf{r}_{\ell} \\
\vdots \\
e_{m}^{(\ell)} \mathbf{r}_{\ell}
\end{array}\right) \in \mathbb{R}^{m n}
$$

are linearly independent. This is not difficult; for example, by taking $\mathbf{e}^{(\ell)}=\hat{\mathbf{x}}_{j}, k_{\ell}=$ $\ell-j n, \ell=1, \ldots, m n$ with $j=j(\ell)$, determined from $(j-1) n \leq \ell<j n$, as will be illustrated in example 2.1.

Now we can find the constants $\alpha_{\ell} \in \mathbb{R}, \quad \ell=1, \ldots, m n$ such that:

$$
\sum_{\ell=1}^{m n} \alpha_{\ell} \boldsymbol{\Phi}_{\ell}=\boldsymbol{\Phi}
$$

Using the previous definitions we can rewrite $\left(t-t_{0}\right) \mathbf{u}_{t}\left(\mathbf{x}_{0}, t_{0}\right)$ and $\sum_{j}^{m}\left(x_{j}-x_{0 j}\right) \mathbf{u}_{x_{i}}\left(\mathbf{x}_{0}, t_{0}\right)$ as follows:

$$
\begin{aligned}
\left(t-t_{0}\right) \mathbf{u}_{t}\left(\mathbf{x}_{0}, t_{0}\right) & =-\left(t-t_{0}\right) \sum_{j=1}^{m} A_{j} \mathbf{u}_{x_{j}}\left(\mathbf{x}_{0}, t_{0}\right) \\
& =-\left(t-t_{0}\right) \sum_{j=1}^{m} A_{j} \sum_{\ell=1}^{m n} \alpha_{\ell} e_{j}^{(\ell)} \mathbf{r}_{\ell} \\
& =-\left(t-t_{0}\right) \sum_{\ell=1}^{m n} \alpha_{\ell}\left(\sum_{j=1}^{m} e_{j}^{(\ell)} A_{j} \mathbf{r}_{\ell}\right) \\
& =-\left(t-t_{0}\right) \sum_{\ell=1}^{m n} \alpha_{\ell}\left(\sum_{j=1}^{m} \lambda_{\ell} \mathbf{r}_{\ell}\right)
\end{aligned}
$$

and

$$
\sum_{j=1}^{m}\left(x_{j}-x_{0 j}\right) \mathbf{u}_{x_{j}}\left(\mathbf{x}_{0}, t_{0}\right)=\sum_{\ell=1}^{m n} \alpha_{\ell}\left(\sum_{j=1}^{m} e_{j}^{(\ell)} \mathbf{r}_{\ell}\left(x_{j}-x_{0 j}\right)\right) .
$$

Therefore (2.1) can be written as

$$
\begin{aligned}
\mathbf{u}(\mathbf{x}, t) & =\mathbf{u}\left(\mathbf{x}_{0}, t_{0}\right)+\left(t-t_{0}\right) \mathbf{u}_{t}\left(\mathbf{x}_{0}, t_{0}\right)+\sum_{j=1}^{m}\left(x_{j}-x_{0 j}\right) \mathbf{u}_{x_{j}}\left(\mathbf{x}_{0}, t_{0}\right)+o\left(\left|\mathbf{x}-\mathbf{x}_{0}\right|+\left|t-t_{0}\right|\right) \\
& =\mathbf{u}\left(\mathbf{x}_{0}, t_{0}\right)+\sum_{\ell=1}^{m n} \alpha_{\ell} \mathbf{r}_{\ell}\left[\mathbf{e}^{(\ell)} \cdot\left(\mathbf{x}-\mathbf{x}_{0}\right)-\lambda_{\ell}\left(t-t_{0}\right)\right]+o\left(\left|\mathbf{x}-\mathbf{x}_{0}\right|+\left|t-t_{0}\right|\right) .
\end{aligned}
$$

We can now define a basis of wave solutions, $\left\{\mathbf{u}_{\mathbf{e}^{(\ell)}}\right\}$, independent of $\mathbf{u}$, but determined by the specific choice of $\left\{\mathbf{e}^{(\ell)}\right\}$, as

$$
\mathbf{u}_{\mathbf{e}^{(\ell)}}=\mathbf{r}_{\ell}\left[\mathbf{e}^{(\ell)} \cdot\left(\mathbf{x}-\mathbf{x}_{0}\right)-\lambda_{\ell}\left(t-t_{0}\right)\right] .
$$


Note that each $\mathbf{u}_{\mathbf{e}^{(\ell)}}$ is a solution of $(1.7)$ and is a wave moving in the direction $\left(\mathbf{e}^{(\ell)}\right)$, or opposite to $\left(\mathbf{e}^{(\ell)}\right)$. Then $(2.1)$ becomes:

$$
\mathbf{u}(\mathbf{x}, t)=\mathbf{u}\left(\mathbf{x}_{0}, t_{0}\right)+\sum_{\ell=1}^{m n} \alpha_{\ell} \mathbf{u}_{\mathbf{e}^{(\ell)}}+o\left(\left|\mathbf{x}-\mathbf{x}_{0}\right|+\left|t-t_{0}\right|\right),
$$

i.e., the first-order term in the Taylor expansion of $\mathbf{u}(\mathbf{x}, t)$ can be presented as a linear combination of the linear waves $\mathbf{u}_{\mathbf{e}}(\ell)$.

Also, higher-order terms in $\mathbf{u}$ can be similarly expanded, obtaining an expression

$$
\mathbf{u}(\mathbf{x}, t)=\mathbf{u}\left(\mathbf{x}_{0}, t_{0}\right)+\sum_{\kappa=1}^{p} \sum_{\ell_{\kappa}=1}^{p_{\kappa}} \alpha_{\ell_{\kappa}} \mathbf{u}_{\mathbf{e}^{\left(\ell_{\kappa}\right)}, \kappa}+o\left(\left|\mathbf{x}-\mathbf{x}_{0}\right|^{p}+\left|t-t_{0}\right|^{p}\right)
$$

for $\mathbf{u}$ locally sufficiently smooth, with

$$
\mathbf{u}_{\mathbf{e}^{(\ell)}, \kappa}=\mathbf{r}_{\ell}\left[\mathbf{e}^{(\ell)} \cdot\left(\mathbf{x}-\mathbf{x}_{0}\right)-\lambda_{k_{\ell}}\left(t-t_{0}\right)\right]^{\kappa}
$$

and $\mathbf{r}_{\ell}$ and $\lambda_{k_{\ell}}$ satisfying (1.9). We omit the proof of this in the interest of brevity.

For $\boldsymbol{\nu}$ the outward unit normal at $\mathbf{x}_{0}, \mathbf{e} \in \mathbb{R}^{m} / \mathbf{0}$ and $\lambda$ determined from (1.9), we denote the set of outgoing, incoming, and stationary or tangential waves in the neighborhood of $\mathbf{x}_{0}$ by

$$
\begin{array}{ll}
\mathcal{U}_{+}=\left\{\mathbf{u}_{\mathbf{e}} \mid(\mathbf{e} \cdot \boldsymbol{\nu}) \lambda>0\right\} & \text { outgoing, } \\
\mathcal{U}_{-}=\left\{\mathbf{u}_{\mathbf{e}} \mid(\mathbf{e} \cdot \boldsymbol{\nu}) \lambda<0\right\} & \text { incoming, } \\
\mathcal{U}_{0}=\left\{\mathbf{u}_{\mathbf{e}} \mid(\mathbf{e} \cdot \boldsymbol{\nu}) \lambda=0\right\} & \text { stationary or tangential. }
\end{array}
$$

We also define the following subspaces of local solutions of (1.7):

$$
\begin{aligned}
& \hat{\mathcal{U}}_{+}=\operatorname{span}\left\{\mathbf{u}_{\mathbf{e}} \in \mathcal{U}_{+}\right\}, \\
& \hat{\mathcal{U}}_{-}=\operatorname{span}\left\{\mathbf{u}_{\mathbf{e}} \in \mathcal{U}_{-}\right\}, \\
& \hat{\mathcal{U}}_{0}=\operatorname{span}\left\{\mathbf{u}_{\mathbf{e}} \in \mathcal{U}_{0}\right\},
\end{aligned}
$$

and their equivalents in $\mathbb{R}^{m n}$ :

$$
\begin{aligned}
& \tilde{\mathcal{U}}_{+}=\operatorname{span}\left\{\boldsymbol{\Phi}_{\ell} \mid \mathbf{u}_{\mathbf{e}} \in \mathcal{U}_{+}\right\}, \\
& \tilde{\mathcal{U}}_{-}=\operatorname{span}\left\{\boldsymbol{\Phi}_{\ell} \mid \mathbf{u}_{\mathbf{e}} \in \mathcal{U}_{-}\right\}, \\
& \tilde{\mathcal{U}}_{0}=\operatorname{span}\left\{\boldsymbol{\Phi}_{\ell} \mid \mathbf{u}_{\mathbf{e}} \in \mathcal{U}_{0}\right\} .
\end{aligned}
$$

Similarly, for a given choice of the vectors $\mathbf{e}^{(\ell)}$ and indices $k_{\ell}$, the waves $\mathbf{u}_{\mathbf{e}^{(\ell)}}$ can now be characterized as follows:

$$
\begin{array}{ll}
W_{+}=\left\{\mathbf{u}_{\mathbf{e}^{(\ell)}} \mid\left(\mathbf{e}^{(\ell)} \cdot \boldsymbol{\nu}\right) \lambda_{\ell}>0\right\} & \text { outgoing, } \\
W_{-}=\left\{\mathbf{u}_{\mathbf{e}^{(\ell)}} \mid\left(\mathbf{e}^{(\ell)} \cdot \boldsymbol{\nu}\right) \lambda_{\ell}<0\right\} & \text { incoming, } \\
W_{0}=\left\{\mathbf{u}_{\mathbf{e}^{(\ell)}} \mid\left(\mathbf{e}^{(\ell)} \cdot \boldsymbol{\nu}\right) \lambda_{\ell}=0\right\} & \text { stationary or tangential. }
\end{array}
$$


We define the corresponding subspaces:

$$
\begin{aligned}
& \hat{W}_{+}=\operatorname{span}\left\{\mathbf{u}_{\mathbf{e}^{(\ell)}} \in W_{+}\right\}, \\
& \hat{W}_{-}=\operatorname{span}\left\{\mathbf{u}_{\mathbf{e}^{(\ell)}} \in W_{-}\right\}, \\
& \hat{W}_{0}=\operatorname{span}\left\{\mathbf{u}_{\mathbf{e}^{(\ell)}} \in W_{0}\right\},
\end{aligned}
$$

and their equivalents in $\mathbb{R}^{m n}$ :

$$
\begin{aligned}
& \tilde{W}_{+}=\operatorname{span}\left\{\boldsymbol{\Phi}_{\ell} \mid \mathbf{u}_{\mathbf{e}^{(\ell)}} \in W_{+}\right\} \\
& \tilde{W}_{-}=\operatorname{span}\left\{\boldsymbol{\Phi}_{\ell} \mid \mathbf{u}_{\mathbf{e}^{(\ell)}} \in W_{-}\right\}, \\
& \tilde{W}_{0}=\operatorname{span}\left\{\boldsymbol{\Phi}_{\ell} \mid \mathbf{u}_{\mathbf{e}^{(\ell)}} \in W_{0}\right\} .
\end{aligned}
$$

Throughout we take $m n$ vectors $\mathbf{e}^{(\ell)}$ and indices $k_{\ell}$ such that the $\mathbf{u}_{\mathbf{e}^{(\ell)}}$ are linearly independent. Therefore the spaces $\hat{W}_{+}, \hat{W}_{-}$, and $\hat{W}_{0}$ are not intersecting. Though it is clear as to which of the $\mathbf{u}_{\mathbf{e}^{(\ell)}}$ are incoming or outgoing, in general, it is not true that $\tilde{W}_{+}\left(\right.$or $\left.\hat{W}_{+}\right)$contains only outgoing waves!

In order to illustrate the phenomenon, let us look again at (1.10) in the domain $\Omega=\{(x, y) \mid x<0\}$. The point $\mathbf{x}_{0}$ will be taken as $\mathbf{x}_{0}=\left(x_{0}, y_{0}\right)=(0,0)$, and the outward unit normal is $\boldsymbol{\nu}=(1,0)$.

The vector $\boldsymbol{\Phi}$ is obtained from (2.2):

$$
\boldsymbol{\Phi}=\left(\begin{array}{c}
\mathbf{u}_{x}\left(\mathbf{x}_{0}, t_{0}\right) \\
\mathbf{u}_{y}\left(\mathbf{x}_{0}, t_{0}\right)
\end{array}\right)=\left(\begin{array}{c}
\partial / \partial x u_{1}\left(\mathbf{x}_{0}, t_{0}\right) \\
\partial / \partial x u_{2}\left(\mathbf{x}_{0}, t_{0}\right) \\
\partial / \partial x u_{3}\left(\mathbf{x}_{0}, t_{0}\right) \\
\partial / \partial y u_{1}\left(\mathbf{x}_{0}, t_{0}\right) \\
\partial / \partial y u_{2}\left(\mathbf{x}_{0}, t_{0}\right) \\
\partial / \partial y u_{3}\left(\mathbf{x}_{0}, t_{0}\right)
\end{array}\right) \in \mathbb{R}^{6}
$$

There are several ways to choose the vectors $\mathbf{e}^{(\ell)}$.

EXAMPLE 2.1.

$$
\mathbf{e}^{(1)}=\mathbf{e}^{(2)}=\mathbf{e}^{(3)}=\left(\begin{array}{l}
1 \\
0
\end{array}\right) ; \quad \mathbf{e}^{(4)}=\mathbf{e}^{(5)}=\mathbf{e}^{(6)}=\left(\begin{array}{l}
0 \\
1
\end{array}\right) .
$$

The corresponding $\lambda_{\ell}$ and $\mathbf{r}_{\ell}$ are

$$
\begin{aligned}
& \lambda_{1}=-1, \quad \mathbf{r}_{1}=\left(\begin{array}{l}
0 \\
1 \\
1
\end{array}\right) ; \quad \lambda_{4}=-1, \quad \mathbf{r}_{4}=\left(\begin{array}{c}
1 \\
0 \\
1
\end{array}\right) ; \\
& \lambda_{2}=0, \quad \mathbf{r}_{2}=\left(\begin{array}{l}
1 \\
0 \\
0
\end{array}\right) ; \quad \lambda_{5}=0, \quad \mathbf{r}_{5}=\left(\begin{array}{c}
0 \\
1 \\
0
\end{array}\right) ; \\
& \lambda_{3}=1, \quad \mathbf{r}_{3}=\left(\begin{array}{r}
0 \\
-1 \\
1
\end{array}\right) ; \quad \lambda_{6}=1, \quad \mathbf{r}_{6}=\left(\begin{array}{r}
-1 \\
0 \\
1
\end{array}\right)
\end{aligned}
$$


The $\boldsymbol{\Phi}_{\ell}$ 's are:

$$
\begin{aligned}
& \boldsymbol{\Phi}_{1}=\left(\begin{array}{c}
0 \\
1 \\
1 \\
0 \\
0 \\
0
\end{array}\right) ; \quad \boldsymbol{\Phi}_{2}=\left(\begin{array}{c}
1 \\
0 \\
0 \\
0 \\
0 \\
0
\end{array}\right) ; \quad \boldsymbol{\Phi}_{3}=\left(\begin{array}{r}
0 \\
-1 \\
1 \\
0 \\
0 \\
0
\end{array}\right) \\
& \boldsymbol{\Phi}_{4}=\left(\begin{array}{c}
0 \\
0 \\
0 \\
1 \\
0 \\
1
\end{array}\right) ; \quad \boldsymbol{\Phi}_{5}=\left(\begin{array}{c}
0 \\
0 \\
0 \\
0 \\
1 \\
0
\end{array}\right) ; \quad \boldsymbol{\Phi}_{6}=\left(\begin{array}{r}
0 \\
0 \\
0 \\
-1 \\
0 \\
1
\end{array}\right) ;
\end{aligned}
$$

and the $\mathbf{u}_{\mathbf{e}^{(\ell)}}$ 's are:

$$
\begin{aligned}
& \mathbf{u}_{\mathbf{e}^{(1)}}=\left(\begin{array}{l}
0 \\
1 \\
1
\end{array}\right)(x+t) ; \quad \mathbf{u}_{\mathbf{e}^{(2)}}=\left(\begin{array}{l}
1 \\
0 \\
0
\end{array}\right) x ; \quad \mathbf{u}_{\mathbf{e}^{(3)}}=\left(\begin{array}{r}
0 \\
-1 \\
1
\end{array}\right)(x-t) ; \\
& \mathbf{u}_{\mathbf{e}^{(4)}}=\left(\begin{array}{l}
1 \\
0 \\
1
\end{array}\right)(y+t) ; \quad \mathbf{u}_{\mathbf{e}^{(5)}}=\left(\begin{array}{l}
0 \\
1 \\
0
\end{array}\right) y ; \quad \mathbf{u}_{\mathbf{e}^{(6)}}=\left(\begin{array}{r}
-1 \\
0 \\
1
\end{array}\right)(y-t) .
\end{aligned}
$$

Here $\mathbf{u}_{\mathbf{e}^{(1)}}$ is an incoming, or left-going wave, $\mathbf{u}_{\mathbf{e}^{(3)}}$ is an outgoing, or right-going wave, $\mathbf{u}_{\mathbf{e}^{(2)}}$ and $\mathbf{u}_{\mathbf{e}^{(5)}}$ are stationary waves, and $\mathbf{u}_{\mathbf{e}^{(4)}}$ and $\mathbf{u}_{\mathbf{e}^{(6)}}$ are tangential waves, i.e., $\mathbf{u}_{\mathbf{e}^{(1)}} \in$ $W_{-}, \mathbf{u}_{\mathbf{e}^{(3)}} \in W_{+}$, and $\mathbf{u}_{\mathbf{e}^{(2)}}, \mathbf{u}_{\mathbf{e}^{(4)}}, \mathbf{u}_{\mathbf{e}^{(5)}}, \mathbf{u}_{\mathbf{e}^{(6)}} \in W_{0}$.

In this case, the dimension of $\tilde{W}_{+}$is 1 , and no input wave can be expanded as a linear combination of vectors from $\tilde{W}_{+}$and $\tilde{W}_{0}$ only.

In Example 1.1, however, $\mathbf{u}_{\mathbf{e}^{(1)}}, \ldots, \mathbf{u}_{\mathbf{e}^{(5)}} \in W_{+}$. Their linear combination, (1.13), is in $W_{-}$.

Thus in the neighborhood of a given point $\mathbf{x}_{0}, t_{0}$ a solution $\mathbf{u}$ can be approximated from a finite-dimensional set of polynomial waves $\mathbf{u}_{\mathbf{e}^{(\ell)}}$. This is in contrast to the situation for trigonometric polynomial waves. It is well known that trigonometric polynomial waves travelling in different directions are orthogonal to each other.

3. The general theorem. In this section it will be proven that the solutions of all hyperbolic systems that couple the outgoing and incoming waves and have no stationary or tangential waves (along the boundary $\partial \Omega$ ) have the phenomena that the incoming and outgoing waves are not separate subspaces, i.e., $\hat{\mathcal{U}}_{+} \cap \hat{\mathcal{U}}_{-} \neq \mathbf{0}$. It should be noted that this theorem holds at any point $\mathbf{x}_{0} \in \partial \Omega$ where these hypotheses are satisfied. We start by proving the theorem for linear waves. Then we briefly sketch the proof for the case when $f$ in (1.8) is a higher-order polynomial. 
3.1. Proof of the general theorem, linear case. Let

$$
\mathbf{u}_{t}+\sum_{i=1}^{m} A_{i} \mathbf{u}_{x_{i}}=\mathbf{0}, \quad \mathbf{x} \in \Omega \subset \mathbb{R}^{m}, \quad t>0, \quad \mathbf{u}(\mathbf{x}, t) \in \mathbb{R}^{n}, \quad m \geq 2,
$$

where

$$
A_{i} \in M_{n \times n} \quad \text { symmetric, } \quad i=1, \ldots, m
$$

and let $\Omega \subset \mathbb{R}^{m}$ be a given domain, with smooth $\partial \Omega, \mathbf{x}_{0} \in \partial \Omega$ and $\boldsymbol{\nu}$ is an outward unit normal at $\mathbf{x}_{0}$.

The system (3.1) has local linear plane wave solutions

$$
\mathbf{u}_{\mathbf{e}(k)}(\mathbf{x}, t)=\mathbf{r}_{k}(\mathbf{e})\left(\mathbf{e} \cdot \mathbf{x}-\lambda_{k}(\mathbf{e}) t\right),
$$

where $\mathbf{e} \in \mathbb{R}^{m} / \mathbf{0}, k \in\{1, \ldots, n\}, \mathbf{r}_{k}(\mathbf{e}) \in \mathbb{R}^{n} / \mathbf{0}$, and $\lambda_{k}$ is defined by

$$
(\mathbf{e} \cdot \mathbf{A}) \mathbf{r}_{k}(\mathbf{e})=\lambda_{k}(\mathbf{e}) \mathbf{r}_{k}(\mathbf{e}) .
$$

Within a neighborhood of a point $\mathbf{x}_{0} \in \partial \Omega$, we have the classification of the plane waves $\mathcal{U}_{+}, \mathcal{U}_{-}$, and $\mathcal{U}_{0}$, as defined in (2.15), and the corresponding subspaces, (2.16)(2.17).

\section{TheOREM 3.1. Assume}

(1) Hypothesis 1: $\partial \Omega$ is non-characteristic at $\mathbf{x}_{0}$ and is smooth in the neighborhood of $\mathbf{x}_{0}$.

(2) Hypothesis 2: There exist $\boldsymbol{\rho}_{ \pm} \in \mathbb{R}^{n} /\{\mathbf{0}\}, \eta_{-}<0<\eta_{+}, \boldsymbol{\mu} \perp \boldsymbol{\nu}$ such that:

$$
(\boldsymbol{\nu} \cdot \mathbf{A}) \boldsymbol{\rho}_{ \pm}=\eta_{ \pm} \boldsymbol{\rho}_{ \pm}
$$

and

$$
\boldsymbol{\rho}_{-}(\boldsymbol{\mu} \cdot \mathbf{A}) \boldsymbol{\rho}_{+}=1
$$

Then

$$
\left.\hat{\mathcal{U}}_{+} \cap \hat{\mathcal{U}}_{-} \neq \mathbf{0} \text { (or, equivalently, } \tilde{\mathcal{U}}_{+} \cap \tilde{\mathcal{U}}_{-} \neq \mathbf{0}\right) \text {. }
$$

Remarks. (1) For a system $\mathbf{u}_{t}+\sum_{i=1}^{m} A_{i} \mathbf{u}_{x_{i}}+K \mathbf{u}=\mathbf{0}, K \in M_{n \times n}(\mathbb{R})$, the same theorem holds, since the zero order term can be eliminated by using the transformation

$$
\mathbf{u}_{\mathbf{e}(k)}(\mathbf{x}, t)=\left(\mathbf{e} \cdot \mathbf{x}-\lambda_{k} t\right) e^{-K t} \mathbf{r}_{k}(\mathbf{e}) .
$$

(2) Hypothesis 1 is not satisfied for a two-dimensional wave equation, (1.10). This theorem does apply, however, to equations like the Advected-Acoustics equations:

$\mathbf{u}_{t}+\left(\begin{array}{ccc}\alpha & 0 & 0 \\ 0 & \alpha & -1 \\ 0 & -1 & \alpha\end{array}\right) \mathbf{u}_{x}+\left(\begin{array}{ccc}\beta & 0 & -1 \\ 0 & \beta & 0 \\ -1 & 0 & \beta\end{array}\right) \mathbf{u}_{y}=\mathbf{0} ; \quad \alpha^{2}+\beta^{2}<1$.

Hypothesis 1 is made in the interest of expediency and can be weakened somewhat. As could be seen from the examples given in the previous sections, the conclusion (3.7) applies in some cases where the boundary is characteristic.

(3) Hypothesis 2, however, is essential. This assumption prevents the case of purely incoming or outgoing boundary, or a combination of two uncoupled systems with boundaries, purely incoming for one and outgoing for the other. 
The proof of theorem 3.1 for linear waves contains two parts. In the first, it is shown that the space of all linear wave solutions to (3.1) is isomorphic to $\mathbb{R}^{m n}$ and that this space can be spanned by all the linear wave solutions moving in two different directions. In the second part it is proven that the incoming and outgoing waves are coupled.

Proof of Theorem 3.1. It suffices to consider $m=2$, with

$$
\mathbf{e}(\theta)=\boldsymbol{\nu}+\theta \boldsymbol{\mu}, \quad \theta \in \mathbb{R} ; \mathbf{e}(\theta) \cdot \boldsymbol{\nu}=1
$$

and let $\lambda_{k}(\theta)$ and $\mathbf{r}_{k}(\theta)$ be determined by (3.4):

$$
(\mathbf{e}(\theta) \cdot \mathbf{A}) \mathbf{r}_{k}(\theta)=\lambda_{k}(\theta) \mathbf{r}_{k}(\theta), \quad k=1, \ldots, n .
$$

From hypothesis 1 , since there are no stationary waves, there exists $\hat{\theta}>0$ such that

$$
\lambda_{k}(\theta) \neq 0,|\theta|<\hat{\theta}, \quad k=1, \ldots, n .
$$

From now on, we shall assume that $|\theta|<\hat{\theta}$.

The claim (3.7) is equivalent to the existence of $\gamma_{j} \in \mathbb{R}, \theta_{j}, k_{j} \in\{1, \ldots, n\}, j=$ $1, \ldots, N$, such that

$$
\sum_{\substack{j=1 \\ \lambda_{j}>0}}^{N^{\prime}} \gamma_{j} \mathbf{u}_{\mathbf{e}\left(\theta_{j}\right), k_{j}}(\mathbf{x}, t)=-\sum_{\substack{j=N^{\prime}+1 \\ \lambda_{j}<0}}^{N} \gamma_{j} \mathbf{u}_{\mathbf{e}\left(\theta_{j}\right), k_{j}}(\mathbf{x}, t) \neq 0,
$$

where $\lambda_{j} \equiv \lambda_{k_{j}}\left(\theta_{j}\right), \mathbf{r}_{j} \equiv \mathbf{r}_{k_{j}}\left(\theta_{j}\right), 1 \leq N^{\prime}<N$, and

$$
\begin{aligned}
& \mathbf{u}_{\mathbf{e}\left(\theta_{j}\right), k_{j}}(\mathbf{x}, t) \in W_{+} ; j \in\left\{1 \ldots N^{\prime}\right\}, \lambda_{j}>0 \\
& \mathbf{u}_{\mathbf{e}\left(\theta_{j}\right), k_{j}}(\mathbf{x}, t) \in W_{-} ; j \in\left\{N^{\prime}+1 \ldots N\right\}, \lambda_{j}<0 .
\end{aligned}
$$

Equation (3.11) means that there is a nontrivial linear combination of incoming waves, which can be expanded as a linear combination of outgoing waves, and vice versa. For the wave solutions $(3.3),(3.11)$ is equivalent to

$$
\begin{array}{rlrl}
\sum_{j=1}^{N} \gamma_{j} \mathbf{r}_{j} & =0 & & \text { coefficients of } \boldsymbol{\nu} \cdot \mathbf{x}, \\
\sum_{j=1}^{N} \theta_{j} \gamma_{j} \mathbf{r}_{j}=0 & & \text { coefficients of } \boldsymbol{\mu} \cdot \mathbf{x}, \\
\sum_{j=1}^{N} \lambda_{j} \gamma_{j} \mathbf{r}_{j}=0 & \text { coefficients of } t .
\end{array}
$$

In fact (3.15) follows from (3.13), (3.14). This can be seen by substituting (3.8) into (3.9). Then:

$$
\sum_{j=1}^{N} \lambda_{j} \gamma_{j} \mathbf{r}_{j}=\sum_{j=1}^{N} \gamma_{j}\left(\left(\boldsymbol{\nu}+\theta_{j} \boldsymbol{\mu}\right) \cdot \mathbf{A}\right) \mathbf{r}_{j}=\boldsymbol{\nu} \cdot \mathbf{A} \sum_{j=1}^{N} \gamma_{j} \mathbf{r}_{j}+\boldsymbol{\mu} \cdot \mathbf{A} \sum_{j=1}^{N} \theta_{j} \gamma_{j} \mathbf{r}_{j}=0 .
$$

For any $\theta, k$, let

$$
\mathbf{q}(\theta, k)=\left(\begin{array}{c}
\mathbf{r}_{k}(\theta) \\
\theta \mathbf{r}_{k}(\theta)
\end{array}\right) \in \mathbb{R}^{2 n}
$$


Then (3.13), (3.14) is equivalent to

$$
\sum_{j=1}^{N} \gamma_{j} \mathbf{q}\left(\theta_{j}, k_{j}\right)=0,
$$

where hereafter in the proof, we shall use $N=2 n+1$. For

$$
\theta^{(1)} \neq \theta^{(2)}
$$

set

$$
\begin{gathered}
\theta_{j}= \begin{cases}\theta^{(1)}, & j=1, \ldots, n \\
\theta^{(2)}, & j=n+1, \ldots, 2 n\end{cases} \\
k_{j}= \begin{cases}j, & j=1, \ldots, n \\
j-n, & j=n+1, \ldots, 2 n .\end{cases}
\end{gathered}
$$

We abbreviate

$$
\mathbf{q}_{j}\left(\theta^{(1)}, \theta^{(2)}\right)=\mathbf{q}\left(\theta_{j}, k_{j}\right), \quad \lambda_{j}=\lambda_{k_{j}}\left(\theta_{j}\right), \quad \mathbf{r}_{j}=\mathbf{r}_{k_{j}}\left(\theta_{j}\right), \quad j=1, \ldots, 2 n
$$

and $M\left(\theta^{(1)}, \theta^{(2)}\right) \in M_{2 n \times 2 n}(\mathbb{R})$ is the matrix, constructed from the $\mathbf{q}_{j}\left(\theta^{(1)}, \theta^{(2)}\right)$, assembled columnwise.

From $(3.14),(3.18),(3.19),(3.20), M\left(\theta^{(1)}, \theta^{(2)}\right)$ is nonsingular, so for any $\tilde{\theta}, \tilde{k}$ there exists a unique $\gamma\left(\tilde{\theta}, \tilde{k} ; \theta^{(1)}, \theta^{(2)}\right) \in \mathbb{R}^{2 n}$, satisfying

$$
M\left(\theta^{(1)}, \theta^{(2)}\right) \gamma\left(\tilde{\theta}, \tilde{k} ; \theta^{(1)}, \theta^{(2)}\right)=\mathbf{q}(\tilde{\theta}, \tilde{k}) .
$$

A solution of (3.22) implies a solution of (3.17) with $N=2 n+1, \gamma_{2 n+1}=-1$, $\theta_{2 n+1}=\tilde{\theta}, k_{2 n+1}=\tilde{k}$. Thus we have shown that every linear wave can be expanded as a linear combination of $2 n$ waves moving forward or backward in the directions determined by $\theta^{(1)}$ and $\theta^{(2)}$. Up to this point, we have said nothing about the signs of the $\lambda_{k}$.

It remains to show that $\theta^{(1)}, \theta^{(2)}, \tilde{\theta}, \tilde{k}$ can be chosen so that (3.11) holds. Specifically, it remains to show that the span of the outgoing (incoming) waves does not contain just outgoing (incoming) waves.

Denote by

$$
\begin{aligned}
& T_{+}\left(\theta^{(1)}, \theta^{(2)}\right)=\operatorname{span}\left\{\mathbf{q}_{j}\left(\theta^{(1)}, \theta^{(2)}\right) \mid \lambda_{j}>0\right\} \\
& T_{-}\left(\theta^{(1)}, \theta^{(2)}\right)=\operatorname{span}\left\{\mathbf{q}_{j}\left(\theta^{(1)}, \theta^{(2)}\right) \mid \lambda_{j}<0\right\} .
\end{aligned}
$$

$T_{+}\left(T_{-}\right)$is the span of all the outgoing (incoming) $\mathbf{q}_{j}\left(\theta^{(1)}, \theta^{(2)}\right)$.

$$
\begin{aligned}
& Y_{+}\left(\theta^{(1)}, \theta^{(2)}\right)=\operatorname{span}_{\tilde{\theta}, \tilde{k}}\left\{\gamma_{j}\left(\tilde{\theta}, \tilde{k} ; \theta^{(1)}, \theta^{(2)}\right) \mathbf{q}_{j}\left(\theta^{(1)}, \theta^{(2)}\right) \mid \lambda_{\tilde{k}}(\tilde{\theta})>0\right\}, \\
& Y_{-}\left(\theta^{(1)}, \theta^{(2)}\right)=\operatorname{span}_{\tilde{\theta}, \tilde{k}}\left\{\gamma_{j}\left(\tilde{\theta}, \tilde{k} ; \theta^{(1)}, \theta^{(2)}\right) \mathbf{q}_{j}\left(\theta^{(1)}, \theta^{(2)}\right) \mid \lambda_{\tilde{k}}(\tilde{\theta})<0\right\},
\end{aligned}
$$

where $\underset{\tilde{\theta}}{\operatorname{span}} \tilde{k}$ means the span over all values of $\tilde{\theta}, \tilde{k} . Y_{+}$(respectively $Y_{-}$) is the span of the $\mathbf{q}_{j}\left(\theta^{(1)}, \theta^{(2)}\right)$ appearing with nonzero coefficients in the solution of $(3.22)$ with $\mathbf{q}(\tilde{\theta}, \tilde{k})$ corresponding to an outgoing (respectively incoming) wave. 


$$
\begin{aligned}
& Z_{+}=\operatorname{span}_{\theta, k}\left\{\mathbf{q}(\theta, k) \mid \lambda_{k}(\theta)>0\right\}, \\
& Z_{-}=\operatorname{span}_{\theta, k}\left\{\mathbf{q}(\theta, k) \mid \lambda_{k}(\theta)<0\right\} .
\end{aligned}
$$

$Z_{+}\left(Z_{-}\right)$is the span of all the outgoing (incoming) $\mathbf{q}(\theta, k)$.

Note that for any $\theta^{(1)}, \theta^{(2)},\left\{\mathbf{q}_{j}\left(\theta^{(1)}, \theta^{(2)}\right) \mid \lambda_{j}>0\right\}\left(\left\{\mathbf{q}_{j}\left(\theta^{(1)}, \theta^{(2)}\right) \mid \lambda_{j}<0\right\}\right)$ is an outgoing (incoming) wave, therefore it is in $Z_{+}\left(Z_{-}\right)$. Thus $T_{ \pm}\left(\theta^{(1)}, \theta^{(2)}\right) \subseteq Z_{ \pm}$. Furthermore, using (3.22), and choosing $\tilde{\theta}=\theta, \tilde{k}=k$, it follows that $Z_{ \pm} \subseteq Y_{ \pm}\left(\theta^{(1)}, \theta^{(2)}\right)$, and therefore

$$
T_{ \pm}\left(\theta^{(1)}, \theta^{(2)}\right) \subseteq Z_{ \pm} \subseteq Y_{ \pm}\left(\theta^{(1)}, \theta^{(2)}\right) .
$$

There are now two possibilities. In the first one $Y_{+}\left(\theta^{(1)}, \theta^{(2)}\right) \nsubseteq T_{+}\left(\theta^{(1)}, \theta^{(2)}\right)$; i.e., there is at least one $\mathbf{q}(\tilde{\theta}, \tilde{k}) \in Y_{+}\left(\theta^{(1)}, \theta^{(2)}\right)$, but $\mathbf{q}(\tilde{\theta}, \tilde{k}) \notin T_{+}\left(\theta^{(1)}, \theta^{(2)}\right)$. This is case 1 below, which we shall show immediately implies the theorem. The other possibility is that

$$
T_{ \pm}\left(\theta^{(1)}, \theta^{(2)}\right)=Z_{ \pm}=Y_{ \pm}\left(\theta^{(1)}, \theta^{(2)}\right) ;
$$

this is case 2 below, which we shall show precluded by hypothesis 2 .

(1) Case 1: Suppose that for some $\theta^{(1)}, \theta^{(2)}$

$$
Y_{+}\left(\theta^{(1)}, \theta^{(2)}\right) \nsubseteq T_{+}\left(\theta^{(1)}, \theta^{(2)}\right)
$$

(or similarly, $Y_{-}\left(\theta^{(1)}, \theta^{(2)}\right) \not \subset T_{-}\left(\theta^{(1)}, \theta^{(2)}\right)$ ).

Then since $Y_{+}\left(\theta^{(1)}, \theta^{(2)}\right) \subset \mathbb{R}^{2 n}$ and $T_{+}\left(\theta^{(1)}, \theta^{(2)}\right) \oplus T_{-}\left(\theta^{(1)}, \theta^{(2)}\right)=\mathbb{R}^{2 n}$, it follows that

$$
Y_{+}\left(\theta^{(1)}, \theta^{(2)}\right) \cap T_{-}\left(\theta^{(1)}, \theta^{(2)}\right) \neq 0 .
$$

From (3.29), there is $\tilde{\theta}, \tilde{k}$ such that $\lambda_{\tilde{k}}(\tilde{\theta})>0$ and such that, for some $j \in$ $\{1, \ldots, 2 n\}, \gamma_{j}\left(\tilde{\theta}, \tilde{k} ; \theta^{(1)}, \theta^{(2)}\right) \neq 0$ and $\lambda_{j}<0$. Since the $\left\{\mathbf{q}_{j}\left(\theta^{(1)}, \theta^{(2)}\right) \mid \lambda_{j}<0\right\}$ are linearly independent, the corresponding solution of $(3.22)$ necessarily satisfies (3.11), thus establishing (3.7).

(2) Case 2: Suppose (3.28) and the corresponding relation between $Y_{-}$and $T_{-}$both fail; then from (3.26), we get (3.27). From (3.27), since $Z_{ \pm}$are independent of $\theta^{(1)}, \theta^{(2)}, T_{ \pm}$are independent of $\theta^{(1)}, \theta^{(2)}$.

Claim: $T_{ \pm}$are independent of $\theta^{(1)}, \theta^{(2)}$ only if

$$
\left.\operatorname{span}_{k}\left\{\mathbf{r}_{k}(\theta) \mid \lambda_{k}(\theta)>0 \text { (or } \lambda_{k}(\theta)<0\right)\right\} \text { is independent of } \theta \text {. }
$$

The proof of the claim is temporarily deferred.

We establish (3.7) by showing that (3.27) is impossible. With $\mathbf{e}(\theta)$ determined from (3.8), fix $k \in\{1, \ldots, n\}$ so that $\lambda_{k}(0)=\eta_{+}$and $\mathbf{r}_{k}(0)=\boldsymbol{\rho}_{+}$as obtained from (3.9) with this particular $k$. Differentiate (3.9) with respect to $\theta$ at $\theta=0$, obtaining

$$
\left(\boldsymbol{\nu} \cdot \mathbf{A}-\eta_{+}\right) \frac{d \mathbf{r}_{k}(0)}{d \theta}+\boldsymbol{\mu} \cdot \mathbf{A} \boldsymbol{\rho}_{+}=\frac{d \lambda_{k}(0)}{d \theta} \boldsymbol{\rho}_{+} .
$$

Using $\boldsymbol{\rho}_{-} \perp \boldsymbol{\rho}_{+}$, as they are eigenvectors of a symmetric matrix, the inner product of (3.31) with $\boldsymbol{\rho}_{-}$gives

$$
\left(\eta_{-}-\eta_{+}\right) \boldsymbol{\rho}_{-} \cdot \frac{d \mathbf{r}_{k}(0)}{d \theta}+\boldsymbol{\rho}_{-} \cdot(\boldsymbol{\mu} \cdot \mathbf{A}) \boldsymbol{\rho}_{+}=0
$$


where $\cdot$ is the standard $\mathbb{R}^{n}$ inner product, so using (3.6) yields

$$
\boldsymbol{\rho}_{-} \cdot \frac{d \mathbf{r}_{k}(0)}{d \theta}=\frac{1}{\eta_{+}-\eta_{-}} .
$$

From (3.33), for $\theta \neq 0$ sufficiently small, $\lambda_{k}(\theta)>0$ and

$$
\boldsymbol{\rho}_{-} \cdot \mathbf{r}_{k}(\theta) \neq \mathbf{0}
$$

contradicting (3.30).

Proof of claim. Denote by $V(\theta)$ the eigenvectors $\left\{\mathbf{r}_{k}(\theta), k=1, \ldots, n \mid \lambda_{k}(\theta)>0\right\}$ assembled columnwise.

From hypothesis 2 , for any $\theta$, there is at least one $\mathbf{r}_{k}(\theta)$ with positive $\lambda_{k}(\theta)$ and at least one with negative $\lambda_{k}(\theta)$. Furthermore, from assumption (1), there is no $\mathbf{r}_{k}(\theta)$ with $\lambda_{k}(\theta)=0$. Since the eigenvalues $\lambda_{k}(\theta)$ are continuous functions of $\theta$, the number of positive and negative $\lambda_{k}(\theta)$ is independent of $\theta$. Thus we have $V(\theta) \in M_{n \times \ell}(\mathbb{R})$ for some $\ell, 1 \leq \ell \leq n-1, \ell$ independent of $\theta$.

Now from (3.23), $T_{+}$independent of $\theta^{(1)}, \theta^{(2)}$ means that

$$
\mathbf{q}(\theta, k) \in T_{+}\left(\theta^{(1)}, \theta^{(2)}\right)
$$

for any $\theta^{(1)}, \theta^{(2)}, \theta, k$ such that $\lambda_{k}(\theta)>0$.

Using (3.16), we have from (3.35) and the definition of $V(\theta)$ the existence of $F, G \in$ $M_{\ell \times \ell}(\mathbb{R})$ (depending on $\left.\theta^{(1)}, \theta^{(2)}, \theta\right)$ such that

$$
V\left(\theta^{(1)}\right) F+V\left(\theta^{(2)}\right) G=V(\theta)
$$

and

$$
\theta^{(1)} V\left(\theta^{(1)}\right) F+\theta^{(2)} V\left(\theta^{(2)}\right) G=\theta V(\theta)
$$

which has to hold for all $\theta^{(1)}, \theta^{(2)}, \theta$.

Choosing $\theta^{(1)}, \theta^{(2)}, \theta$ distinct, we solve (3.36), (3.37) simultaneously, obtaining

$$
V\left(\theta^{(1)}\right) F=\frac{\theta-\theta^{(2)}}{\theta^{(1)}-\theta^{(2)}} V(\theta)
$$

and

From (3.38), (3.39)

$$
V\left(\theta^{(2)}\right) G=\frac{\theta-\theta^{(1)}}{\theta^{(2)}-\theta^{(1)}} V(\theta) .
$$

$$
R(V(\theta)) \subseteq R\left(V\left(\theta^{(1)}\right)\right) \quad \text { or } \quad R\left(V\left(\theta^{(2)}\right)\right) .
$$

But as $\theta^{(1)}, \theta^{(2)}, \theta$ can be interchanged, (3.40) implies

$$
R\left(V\left(\theta^{(1)}\right)\right)=R\left(V\left(\theta^{(2)}\right)\right)=R(V(\theta)),
$$

which is equivalent to (3.30).

Thus case $2,(3.27)$, is precluded. This proves the claim and completes the proof of theorem 3.1 .

If hypothesis 1 holds, then hypothesis 2 is necessary as well as sufficient for theorem 3.1 to hold.

Theorem 3.2. Assume that the $A_{i}, i=1, \ldots, m$, in (3.1) are independent of $\mathbf{x}, t$. Assume

- The boundary $\partial \Omega$ is piecewise smooth and noncharacteristic almost everywhere.

- Hypothesis 2 of theorem 3.1 fails almost everywhere. 
Then the system (3.1) is equivalent to an assembly of uncoupled subsystems, for each of which the boundary is almost everywhere noncharacteristic and completely incoming or else completely outgoing.

REMARKS. (a) In the above, assumption (2) means that at every such point the boundary is completely incoming or completely outgoing so that (3.5) fails or else (3.6) fails; i.e., there is no such vector $\boldsymbol{\mu}$.

(b) This theorem does not survive the addition of a lower-order term in $u$ to the system (3.1).

(c) The extreme case of a system being equivalent to $n$ scalar equations corresponds to the case where the matrices $A_{i}, i=1, \ldots, m$ all commute.

We omit the proof of this theorem in the interest of brevity.

Generalization to higher powers. The theorem above is proven for waves in the form $\mathbf{u}_{\mathbf{e}, k}=\mathbf{r}_{k}(\mathbf{e})\left(\mathbf{e} \cdot \mathbf{x}-\lambda_{k}(\mathbf{e}) t\right)$. It can be shown that the theorem is also true for higher-order polynomial waves, namely $\mathbf{u}_{\mathbf{e}, k, \kappa}=\mathbf{r}_{k}(\mathbf{e})\left(\mathbf{e} \cdot \mathbf{x}-\lambda_{k}(\mathbf{e}) t\right)^{\kappa}, \kappa \in \mathbb{N}$. The proof is similar to the linear case.

4. Absorbing boundary conditions. In this section we discuss the question of nonreflecting boundary conditions as presented in section 1 . Under the same assumptions as for theorem 3.1, we show that local, linear, perfectly nonreflecting boundary conditions do not exist.

4.1. A theorem on boundary conditions.

TheOREM 4.1. Under the same hypothesis as made in theorem 3.1, there is no boundary condition (1.6) sufficient to uniquely determine weak solutions of (1.4) such that the restriction of the solution $\tilde{\mathbf{u}}$ obtained from (1.1) to $\Omega$ coincides with the corresponding solution of (1.4).

REMARK. This theorem applies to linear, local boundary conditions. The questions of nonlinear, nonlocal conditions and absorbing boundary layers (PML) are still open. For more information about the PML boundary conditions, see [3], [4], [1], and [2].

Proof. Throughout the proof, the point $\mathbf{x}_{0} \in \partial \Omega$ is considered fixed; nothing depends on $\mathbf{x}_{0}$ in particular. Hypotheses 1 and 2 are assumed to be satisfied at $\mathbf{x}_{0}$.

For any $\mathbf{x} \in \partial \Omega$, denote by $\boldsymbol{\nu}(\mathbf{x})$ the unit normal at this point.

Let

$$
\begin{gathered}
S(\boldsymbol{\nu}(\mathbf{x}))=\left\{(P, Q), \quad P, Q \in M_{n \times n} \text { symmetric }\right\}, \\
P+Q=\boldsymbol{\nu}(\mathbf{x}) \cdot \mathbf{A}, \\
P \geq 0, \quad Q \leq 0, \\
\mathrm{R}(P) \cap \mathrm{R}(Q)=\mathbf{0} .
\end{gathered}
$$

See [5], [6].

As an example, let $\boldsymbol{\nu}(\mathbf{x}) \cdot \mathbf{A}=\left(\begin{array}{ll}0 & 1 \\ 1 & 0\end{array}\right)$. Then, for any $1 \geq \alpha>0$, we can take

$$
P=\frac{1}{2}\left(\begin{array}{cc}
1 / \alpha & 1 \\
1 & 1 / \alpha
\end{array}\right), \quad Q=\frac{1}{2}\left(\begin{array}{cc}
-1 / \alpha & 1 \\
1 & -1 / \alpha
\end{array}\right) \text {. }
$$


We define $P_{0}$ and $Q_{0}$ as the specific $P$ and $Q$ which also satisfy:

$$
P_{0} Q_{0}=Q_{0} P_{0}=\mathbf{0} \text {. }
$$

In this example, $P_{0}$ and $Q_{0}$ correspond to $\alpha=1$.

In order to prove theorem 4.1, we need the following lemmas:

Lemma 4.2. Given $\omega \subset \mathbb{R}^{m}$ with smooth boundary $\partial \omega$ and outward unit normal $\boldsymbol{\zeta}(\mathbf{x}), \mathbf{x} \in$ $\partial \omega$

$$
P, Q \in S(\boldsymbol{\zeta}(\mathbf{x})), \text { for all } \mathbf{x} \in \partial \omega
$$

and

$$
\begin{aligned}
& \mathbf{z}(\cdot, 0) \in L_{2}(\omega) \\
& \mathbf{g} \in L_{2}(\omega \times(0, T)), \quad 0<T<\infty \\
& \mathbf{b} \in L_{2}(\partial \omega \times(0, T)),
\end{aligned}
$$

there exists a unique solution of

$$
\begin{aligned}
& \mathbf{z}_{t}+\sum_{i=1}^{m} A_{i} \mathbf{z}_{x_{i}}=\mathbf{g}, \quad \mathbf{x} \in \omega, \quad 0<t<T, \\
& Q \mathbf{z}=Q \mathbf{b}, \quad \mathbf{x} \in \partial \omega, \quad 0<t<T \quad\left(\text { weakly, in } L_{2}(\partial \omega \times(0, T)),\right. \\
& \mathbf{z} \in L_{2}(\omega \times(0, T)), \quad P \mathbf{z} \in L_{2}(\partial \omega \times(0, T)) .
\end{aligned}
$$

Lemma 4.2 states that boundary conditions of the form (4.11) suffice for uniqueness, with $\{P, Q\} \in S(\boldsymbol{\nu}(\mathbf{x}))$ for almost all $\mathbf{x} \in \partial \omega$.

We observe that $\omega$ does not have to be bounded.

We defer the proof to section 5 .

Lemma 4.3. Given $\mathbf{x}, \mathbf{x}^{\prime} \in \partial \Omega$,

$$
\{P, Q\} \in S(\boldsymbol{\nu}(\mathbf{x}))
$$

Then there exists

$$
\left\{P^{\prime}, Q^{\prime}\right\} \in S\left(\boldsymbol{\nu}\left(\mathbf{x}^{\prime}\right)\right)
$$

such that

$$
\left\|P-P^{\prime}\right\|,\left\|Q-Q^{\prime}\right\| \leq c\left|\mathbf{x}-\mathbf{x}^{\prime}\right| .
$$

The proof is deferred to section 6 .

Proof of Theorem 4.1. Since $\tilde{\mathbf{u}}$, satisfying (1.1), is unique, the boundary condition $(1.6),\left.B \mathbf{u}\right|_{\partial \Omega}=0$, must imply that $\mathbf{u}$ satisfying (1.4)-(1.6) is uniquely determined. Set $p=\operatorname{rank} P_{0}=$ dimension of positive eigenspace of $\boldsymbol{\nu}\left(\mathbf{x}_{0}\right) \cdot \mathbf{A}$.

From hypothesis 1,

$$
\text { rank } Q_{0}=n-p
$$

so uniqueness of $\mathbf{u}$ can be attained only with $B$ satisfying

$$
\operatorname{rank} B\left(\mathbf{x}_{0}\right) \geq n-p
$$


or

$$
\operatorname{dim} \operatorname{ker} B\left(\mathbf{x}_{0}\right) \leq p
$$

Denote by

$$
X=\left\{\tilde{\mathbf{u}}\left(\mathbf{x}_{0}, t\right), 0<t<T \mid \operatorname{supp} \tilde{\mathbf{u}}(\cdot, 0), \mathbf{g} \in \Omega\right\}
$$

with $\tilde{\mathbf{u}}$ satisfying (1.1).

If $\left.B \mathbf{u}\right|_{\partial \Omega}=\mathbf{0}$ and $\mathbf{u}=\tilde{\mathbf{u}}$ on $\partial \Omega$, then $\left.B \tilde{\mathbf{u}}\right|_{\partial \Omega}=\mathbf{0}$, so to prove the theorem it will suffice to prove

$$
\operatorname{dim} X>p .
$$

The proof of (4.21) follows from three propositions.

Proposition 1. For any $\boldsymbol{\xi} \in \mathbb{R}^{n},(\tilde{P}, \tilde{Q}) \in S\left(\boldsymbol{\nu}\left(\mathbf{x}_{0}\right)\right)$, there exists $\overline{\mathbf{u}} \in X$ satisfying

$$
\tilde{P} \overline{\mathbf{u}}=\tilde{P} \boldsymbol{\xi} .
$$

Proposition 2. If (4.21) fails, i.e., $\operatorname{dim} X \leq p$, then

$$
X=R\left(P_{0}\left(\mathbf{x}_{0}\right)\right) \quad\left(P_{0}, Q_{0}\right) \in S\left(\boldsymbol{\nu}\left(\mathbf{x}_{0}\right)\right)
$$

satisfying (4.5).

Proposition 3. Under assumptions of theorem 3.1, the condition (4.23) is impossible.

Proof of Proposition 1. We apply lemma 4.2, on the existence of weak solutions, with

$$
\omega=\Omega^{c}=\mathbb{R}^{m} / \bar{\Omega} .
$$

We choose

$$
\psi \in H^{1}(\omega) \cap C(\bar{\omega}), \quad \psi\left(\mathbf{x}_{0}\right)=1,
$$

with supp $\psi \cap \partial \Omega$ in a small neighborhood of $\mathbf{x}_{0}$. By appeal to lemma 4.3, we can choose

$$
(P(\mathbf{x}), Q(\mathbf{x})) \in S(\boldsymbol{\nu}(\mathbf{x})), \quad \mathbf{x} \in \partial \Omega \cap \operatorname{supp} \psi(=\partial \omega \cap \operatorname{supp} \psi)
$$

so that

$$
P(\mathbf{x}) \rightarrow P\left(\mathbf{x}_{0}\right)=\tilde{P}, \quad Q(\mathbf{x}) \rightarrow Q\left(\mathbf{x}_{0}\right)=\tilde{Q} \quad \text { as } \quad \mathbf{x} \rightarrow \mathbf{x}_{0} .
$$

Since $\partial \omega=\partial \Omega$ is smooth in a neighborhood of $\mathbf{x}_{0}$, there exists a unique $\mathbf{z} \subset$ $L_{2}(\omega \times(0, T))(T>0$ arbitrary) satisfying

$$
\begin{gathered}
\mathbf{z}_{t}+\sum_{i=1}^{m} A_{i} \mathbf{z}_{x_{i}}=-\sum_{i=1}^{m} \psi_{x_{i}} A_{i} \boldsymbol{\xi}, \quad \mathbf{x} \in \omega, \quad 0<t<T, \\
\mathbf{z}(\cdot, 0)=\mathbf{0}, \\
P(\mathbf{x}) \mathbf{z}(\mathbf{x}, t)=\mathbf{0}, \quad \mathbf{x} \in \partial \omega, \quad 0<t<T .
\end{gathered}
$$

Since

$$
Q \mathbf{z} \in L_{2}(\partial \omega \times(0, T)),
$$

using (4.30) there is an extension of $\mathbf{z}$ to $\Omega$ such that

$$
\mathbf{z} \in H^{1}(\Omega \times(0, T)) .
$$

Then from (4.25), (4.28), (4.29), (4.30),

$$
\overline{\mathbf{u}}(\mathbf{x}, t)=\mathbf{z}(\mathbf{x}, t)+\psi(\mathbf{x}) \boldsymbol{\xi}
$$


satisfies

$$
\overline{\mathbf{u}}_{t}+\sum_{i=1}^{m} A_{i} \overline{\mathbf{u}}_{x_{i}}= \begin{cases}\mathbf{z}_{t}+\sum_{i=1}^{m} A_{i}(\mathbf{z}+\psi \boldsymbol{\xi})_{x_{i}}, & \mathbf{x} \in \Omega \\ \mathbf{0}, & \mathbf{x} \notin \Omega\end{cases}
$$

and we obtain (4.22) from

$$
\tilde{P} \overline{\mathbf{u}}\left(\mathbf{x}_{0}, t\right)=\tilde{P} \mathbf{z}\left(\mathbf{x}_{0}, t\right)+\psi\left(\mathbf{x}_{0}\right) \tilde{P} \boldsymbol{\xi}=\tilde{P} \boldsymbol{\xi}, \quad 0<t<T .
$$

The result (4.35) needs justification, since $\mathbf{z}$ obtained from (4.28)-(4.30) and the existence lemma 4.2 actually satisfy $(4.30)$ in the sense of $L_{2}(\partial \omega)$ but not pointwise. Thus this applies also to $\overline{\mathbf{u}}$ obtained from (4.33) in (4.35).

Given such $\psi$, let $\sigma_{\epsilon}$ be a small open neighborhood of $\mathbf{x}_{0} \in \partial \omega$, shrinking to $\left\{\mathbf{x}_{0}\right\}$ as $\varepsilon \downarrow 0$. Denote by

$$
a_{\varepsilon}=\left(\text { surface area of } \sigma_{\varepsilon}\right)^{1 / 2} .
$$

Then with $P(\mathbf{x}), Q(\mathbf{x})$ satisfying (4.26), (4.27), $\mathbf{z}$ obtained from (4.28)-(4.30), and $\overline{\mathbf{u}}$ obtained from (4.33), we have (4.35) in the $L_{2}(\partial \omega)$ sense, i.e.,

$$
\begin{aligned}
& \frac{1}{a_{\varepsilon}}\|\tilde{P}(\overline{\mathbf{u}}(\mathbf{x}, t))-\boldsymbol{\xi}\|_{L_{2}\left(\sigma_{\varepsilon} \times(0, T)\right)} \\
\leq & \frac{1}{a_{\varepsilon}}\left(\|(\tilde{P}-P(\mathbf{x})) \overline{\mathbf{u}}(\mathbf{x}, t)\|_{L_{2}\left(\sigma_{\varepsilon} \times(0, T)\right)}+\|P(\mathbf{x}) \mathbf{z}(\mathbf{x}, t)\|_{L_{2}\left(\sigma_{\varepsilon} \times(0, T)\right)}\right. \\
& \left.+\|(\psi(\mathbf{x})-1) P(\mathbf{x}) \boldsymbol{\xi}\|_{L_{2}\left(\sigma_{\varepsilon} \times(0, T)\right)}+\|(P(\mathbf{x})-\tilde{P}) \xi\|_{L_{2}\left(\sigma_{\varepsilon} \times(0, T)\right)}\right) .
\end{aligned}
$$

As $\varepsilon \downarrow 0$, using (4.25), (4.15), and (4.31), each term on the right-hand side of $(4.37) \rightarrow 0$.

Proof of Proposition 2. Without loss of generality, we take $\boldsymbol{\nu}\left(\mathbf{x}_{0}\right) \cdot \mathbf{A}$ diagonal, and

$$
P_{0}=\operatorname{diag}\left(\lambda_{1}, \ldots, \lambda_{\mathrm{p}}, 0, \ldots, 0\right), \quad \mathrm{Q}_{0}=\left(0, \ldots, 0, \lambda_{\mathrm{p}+1}, \ldots, \lambda_{\mathrm{n}}\right) .
$$

Choosing $\tilde{P}=P_{0}$ in proposition 1 we have, from (4.22),

$$
P_{0} \mathbb{R}^{n}=\left(\begin{array}{c}
\mathbb{R}^{p} \\
\mathbf{0}_{n-p}
\end{array}\right) \subseteq P_{0} X
$$

where

$$
\mathbf{0}_{n-p}=\left(\begin{array}{c}
0 \\
\vdots \\
0
\end{array}\right) \in \mathbb{R}^{n-p} .
$$

Thus if $\operatorname{dim} X \leq p$, necessarily $\operatorname{dim} X=p$ and

$$
X=\operatorname{span}\left\{\mathbf{h}_{1}, \ldots, \mathbf{h}_{p}\right\}
$$

with

$$
\mathbf{h}_{j} \in \mathbb{R}^{n}, \quad h_{j, i}=\delta_{i j}, \quad i, j \in\{1, \ldots, p\} .
$$

Denote by $H \in M_{n \times p}$ the vectors $\mathbf{h}_{1}, \ldots, \mathbf{h}_{p}$ assembled columnwise.

As proposition 1 holds for any $(\tilde{P}, \tilde{Q}) \in S\left(\boldsymbol{\nu}\left(\mathbf{x}_{0}\right)\right)$, for any $\tilde{P}$ we have

$$
\operatorname{dim} \tilde{P} X=\operatorname{rank} \tilde{P} H=\operatorname{rank} \tilde{P}=p .
$$

The conclusion (4.23) follows from (4.40), provided we show that

$$
h_{j, i}=0, \quad 1 \leq j \leq p, \quad p+1 \leq i \leq n .
$$


Suppose this isn't the case. Then fix $j \in\{1, \ldots, p\}, i \in\{p+1, \ldots, n\}$ such that

$$
h_{j, i} \neq 0 \text {. }
$$

We construct $(\tilde{P}, \tilde{Q}) \in S\left(\boldsymbol{\nu}\left(\mathbf{x}_{0}\right)\right)$ such that (4.42) fails.

For $k, \ell, k^{\prime}, \ell^{\prime} \in\{1, \ldots, n\}$ and for $\alpha>0$, denote by $D_{k, \alpha} \in M_{n \times n}(\mathbb{R})$ the diagonal matrix with components

$$
\left(D_{k, \alpha}\right)_{k^{\prime} \ell^{\prime}}= \begin{cases}1, & k^{\prime}=\ell^{\prime} \neq k \\ \frac{1}{4}(\alpha+1 / \alpha)^{2}, & k^{\prime}=\ell^{\prime}=k \\ 0, & k^{\prime} \neq \ell^{\prime}\end{cases}
$$

and for $k \neq \ell, \beta \neq 0, E_{k, \ell, \beta}$, the "elementary matrix", with components

$$
\left(E_{k, \ell, \beta}\right)_{k^{\prime} \ell^{\prime}}=\delta_{k^{\prime} \ell^{\prime}}+\beta \delta_{k k^{\prime}} \delta_{\ell \ell^{\prime}} .
$$

Claim: For any $\alpha>0$ and any $k, \ell: 1 \leq k \leq p<\ell \leq n$,

$$
\begin{gathered}
\beta=\left|\frac{\lambda_{\ell}}{\lambda_{k}}\right|^{1 / 2}\left(\frac{\alpha-1 / \alpha}{\alpha+1 / \alpha}\right), \quad \beta^{\prime}=\left|\frac{\lambda_{k}}{\lambda_{\ell}}\right|^{1 / 2}\left(\frac{\alpha-1 / \alpha}{\alpha+1 / \alpha}\right), \\
\tilde{P}=E_{k, \ell, \beta} D_{k, \alpha} P_{0} E_{\ell, k, \beta}, \\
\tilde{Q}=E_{\ell, k, \beta^{\prime}} D_{\ell, \alpha} Q_{0} E_{k, \ell, \beta^{\prime}},
\end{gathered}
$$

satisfy $(\tilde{P}, \tilde{Q}) \in S\left(\boldsymbol{\nu}\left(\mathbf{x}_{0}\right)\right)$.

Proof. The pairs $P_{0}$ and $\tilde{P}, Q_{0}$ and $\tilde{Q}$ coincide except for the elements $k k, k \ell, \ell k, \ell \ell$.

From (4.38), (4.45)-(4.49) there are four components, assembled as $2 \times 2$ matrices, of the form

$$
\begin{aligned}
& P_{0}:\left(\begin{array}{cc}
\lambda_{k} & 0 \\
0 & 0
\end{array}\right), \quad Q_{0}:\left(\begin{array}{cc}
0 & 0 \\
0 & \lambda_{\ell}
\end{array}\right), \\
& \tilde{P}: \frac{1}{4}\left(\begin{array}{cc}
\lambda_{k}(\alpha+1 / \alpha)^{2} & \left|\lambda_{k} \lambda_{\ell}\right|^{1 / 2}\left(\alpha^{2}-1 / \alpha^{2}\right) \\
\left|\lambda_{k} \lambda_{\ell}\right|^{1 / 2}\left(\alpha^{2}-\frac{1}{\alpha^{2}}\right) & \left|\lambda_{\ell}\right|\left(\alpha-\frac{1}{\alpha}\right)^{2}
\end{array}\right), \\
& \tilde{Q}: \frac{1}{4}\left(\begin{array}{cc}
-\lambda_{k}(\alpha-1 / \alpha)^{2} & -\left|\lambda_{k} \lambda_{\ell}\right|^{1 / 2}\left(\alpha^{2}-1 / \alpha^{2}\right) \\
-\left|\lambda_{k} \lambda_{\ell}\right|^{1 / 2}\left(\alpha^{2}-\frac{1}{\alpha^{2}}\right) & \lambda_{\ell}(\alpha+1 / \alpha)^{2}
\end{array}\right) .
\end{aligned}
$$

Using (4.50), one easily verifies that $\tilde{P}, \tilde{Q}$ satisfy (4.2)-(4.4). This verifies the claim above.

We set $k=j, \ell=i$ such that (4.44) holds and determines $\beta$ from

$$
\beta=-h_{k, k} / h_{k, \ell}=-1 / h_{k, \ell},
$$

using (4.40). Then $\alpha>0$ is uniquely determined from (4.47) with this value of $\beta$.

Now from (4.48), (4.38), (4.41), (4.46),

$$
P_{0} E_{k, \ell, \beta} \mathbf{h}_{k}=0,
$$

implying

$$
\operatorname{rank} \tilde{P} H \leq p-1,
$$

contradicting (4.42). 
Proof of Proposition 3. We assume hereafter that the theorem fails, and thus by applying proposition $2, \operatorname{dim} X=p$. Take $\tilde{\Omega}$, a small sphere within $\Omega$, such that $\tilde{\mathbf{x}}_{0} \in \partial \tilde{\Omega}$ and $\mathbf{x}_{0} \in \partial \Omega$ are the closest boundary points, and such that

$$
\boldsymbol{\nu}\left(\tilde{\mathbf{x}}_{0}\right)=\boldsymbol{\nu}\left(\mathbf{x}_{0}\right) .
$$

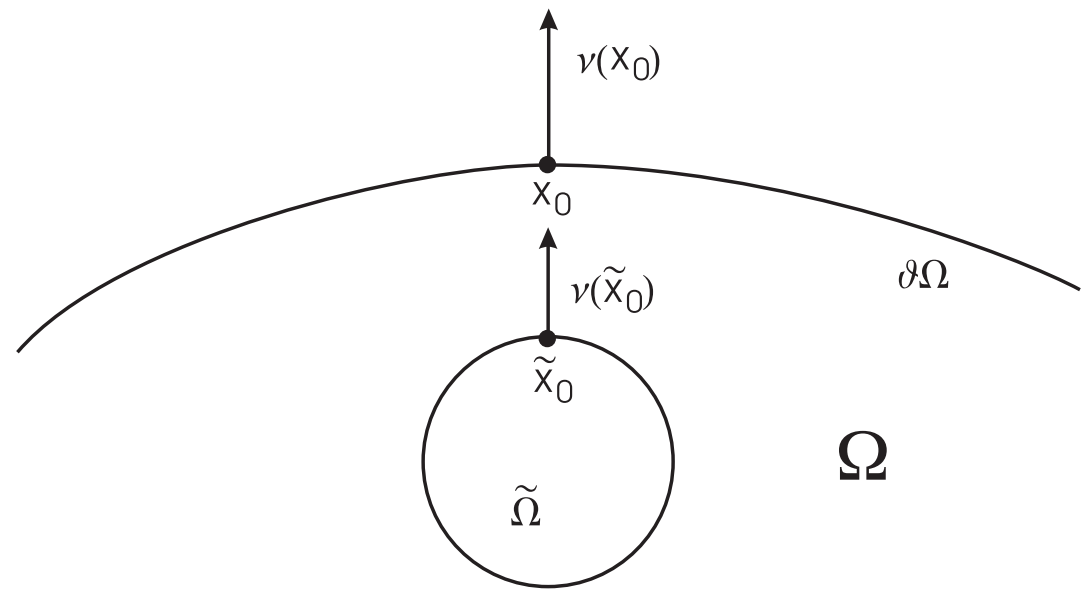

FIG. 1

As hypotheses 1 and 2 of theorem 3.1 apply also to $\tilde{\Omega}$, using (4.54), so do propositions 1 and 2. In particular, using (4.54),

$$
\operatorname{span}\left\{\mathbf{w}\left(\tilde{\mathbf{x}}_{0}, t\right), 0<t<T\right\}=\mathrm{R}\left(P_{0}\left(\boldsymbol{\nu}\left(\tilde{\mathbf{x}}_{0}\right)\right)\right)=\mathrm{R}\left(P_{0}\left(\boldsymbol{\nu}\left(\mathbf{x}_{0}\right)\right)\right),
$$

where the $\mathbf{w}(\mathbf{x}, t)$ satisfy

$$
\mathbf{w}_{t}+\sum_{i=1}^{m} A_{i} \mathbf{w}_{x_{i}}=0, \quad \mathbf{x} \notin \tilde{\Omega}, \quad 0<t<T
$$

and

$$
\mathbf{w}(\mathbf{x}, 0)=\mathbf{0}, \quad \mathbf{x} \notin \tilde{\Omega} .
$$

Choosing $\left|\tilde{\mathbf{x}}_{0}-\mathbf{x}_{0}\right|$ sufficiently small, clearly

$$
\operatorname{dim} \operatorname{span}\left\{\mathbf{w}\left(\mathbf{x}_{0}, t\right), \quad 0<t<T\right\} \geq \operatorname{dim} \operatorname{span}\left\{\mathbf{w}\left(\tilde{\mathbf{x}}_{0}, t\right), \quad 0<t<T\right\},
$$

whence from proposition 2 ,

$$
\operatorname{span}\left\{\mathbf{w}\left(\mathbf{x}_{0}, t\right), \quad 0<t<T\right\}=\mathrm{R}\left(P_{0}\left(\mathbf{x}_{0}\right)\right) .
$$

Now with $\left|\mathbf{x}_{0}-\tilde{\mathbf{x}}_{0}\right|>0$ fixed, there exists $\Omega^{\prime}$ such that

$$
\begin{aligned}
& \tilde{\Omega} \subset \Omega^{\prime}, \\
& \mathbf{x}_{0} \in \partial \Omega^{\prime}, \\
& \boldsymbol{\nu}^{\prime}\left(\mathbf{x}_{0}\right)=\boldsymbol{\nu}\left(\mathbf{x}_{0}\right)+\epsilon \boldsymbol{\mu},
\end{aligned}
$$

with $|\epsilon|>0$ small and $\boldsymbol{\nu}^{\prime}\left(\mathbf{x}_{0}\right)$ is the unit normal to $\partial \Omega^{\prime}$ at $\mathbf{x}_{0}$. 


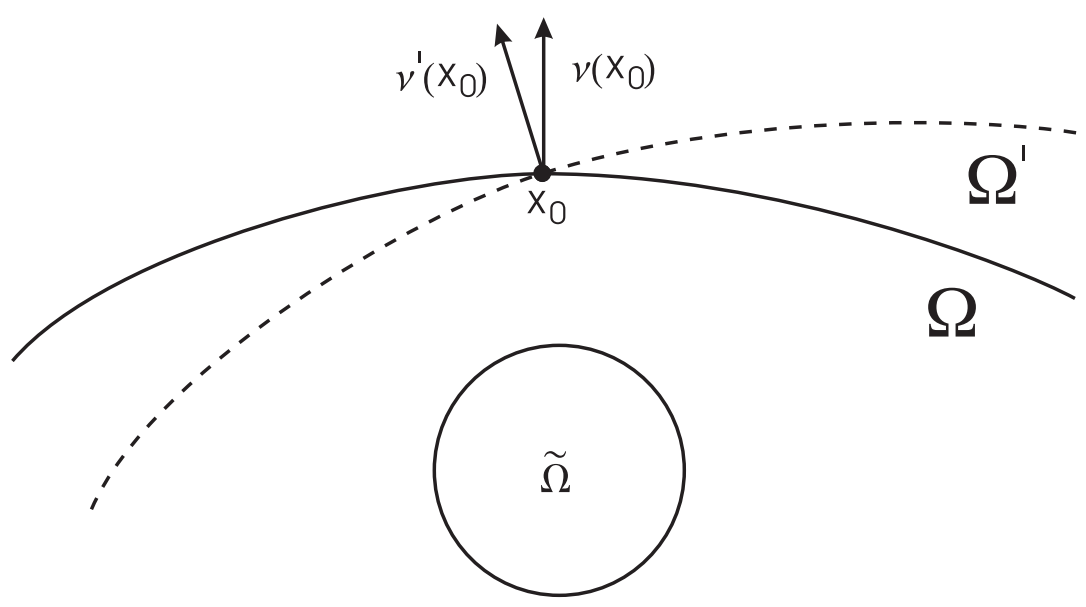

FIG. 2

From (4.60), (4.61),

$$
\left\{\mathbf{w}\left(\mathbf{x}_{0}, t\right)\right\} \subset X^{\prime},
$$

where $X^{\prime}$ is obtained from (4.20), replacing $\Omega$ by $\Omega^{\prime}$. Assumptions (1), (2) and thus propositions 1 and 2 apply also to $\Omega^{\prime}$, so from (4.63)

$$
X^{\prime}=X
$$

and

$$
\mathrm{R}\left(P_{0}^{\prime}\left(\mathbf{x}_{0}\right)\right)=\mathrm{R}\left(P_{0}\left(\mathbf{x}_{0}\right)\right)
$$

and $P_{0}^{\prime}\left(\mathbf{x}_{0}\right)$ is determined using $\boldsymbol{\nu}^{\prime}\left(\mathbf{x}_{0}\right)$.

Now (4.65) is equivalent to (3.30), which has been shown to be impossible under hypothesis 2 .

\section{Proof of Lemma 4.2 .}

Remarks. (1) We allow $A_{i}$ to depend on $\mathbf{x}, t$ with $\left|A_{i}\right|,\left|A_{i, \mathbf{x}_{i}}\right|$ uniformly bounded in $\omega \times(0, T)$.

(2) Adding a term $A_{0} \mathbf{z}$ to the left-hand side of (4.10) with $\left|A_{0}\right|$ (uniformly bounded in $\omega \times(0, T))$ changes nothing.

(3) The issue of boundary conditions for hyperbolic systems has received thorough attention in the literature. In some of the classical textbooks, see for example [7] and [8], the well-posedness is proven by separating the problem into a pure Cauchy problem and a semi-infinite problem for a strip along the boundary, using a partition of unity and a proper change of variable. Here, we use a different approach.

Proof. We take $\left\{H_{N}\right\}_{N=1}^{\infty}$ as a (nested) sequence of subspaces of $H^{1}(\omega) \cap C(\bar{\omega})$ becoming dense in $H^{1}(\omega)$ as $N \rightarrow \infty$. 
Approximations $\mathbf{z}_{N} \in C[0, T] \rightarrow H_{N}$ yield

$$
\begin{gathered}
\mathbf{z}_{N}(0) \longrightarrow \mathbf{z}(\cdot, 0) \text { in } L_{2}(\omega) \text { as } N \rightarrow \infty \\
\int_{\omega}\left[\boldsymbol{\phi} \cdot\left(\mathbf{z}_{N, t}-\mathbf{g}\right)-\mathbf{z}_{N} \cdot \sum_{i=1}^{m}\left(A_{i} \phi\right)_{x_{i}}\right]+\int_{\partial \omega} \phi \cdot\left(Q \mathbf{b}+P \mathbf{z}_{N}\right)=0
\end{gathered}
$$

for all $\phi \in H_{N}, 0<t<T$.

Choose $\phi=\mathbf{z}_{N}$ in (5.2), and integrate with respect to $t$ :

$$
\begin{aligned}
0= & \frac{1}{2} \int_{\omega}\left(\left|\mathbf{z}_{N}(t)\right|^{2}-\left|\mathbf{z}_{N}(0)\right|^{2}\right)-\int_{0}^{t} \int_{\omega} \mathbf{z}_{N} \cdot \mathbf{g} \\
& -\frac{1}{2} \int_{0}^{t} \int_{\omega} \mathbf{z}_{N} \cdot\left(\sum_{i=1}^{m} A_{i, x_{i}}\right) \mathbf{z}_{N}+\int_{0}^{t} \int_{\partial \omega} \mathbf{z}_{N}\left(Q \mathbf{b}+\frac{1}{2}(P-Q) \mathbf{z}_{N}\right), 0<t \leq T
\end{aligned}
$$

So

$$
\left\|\mathbf{z}_{N}(t)\right\|_{L_{2}(w)}, \quad\left\|P^{1 / 2} \mathbf{z}_{N}\right\|_{L_{2}(\partial \omega \times(0, T))}, \quad\left\|(-Q)^{1 / 2} \mathbf{z}_{N}\right\|_{L_{2}(\partial \omega \times(0, T))} \leq C_{T}
$$

uniformly with respect to $N$, using the definition of $(P, Q),(4.2)-(4.4)$.

Extract a weakly convergent subsequence

$$
\begin{array}{rlll}
\stackrel{\mathbf{z}_{N}}{N_{\rightarrow \infty}} \mathbf{z} & \text { in } & L_{2}(\omega \times(0, T)), \\
P^{1 / 2} \mathbf{z}_{N} \stackrel{N \rightarrow \infty}{\longrightarrow} P^{1 / 2} \tilde{\mathbf{z}} & \text { in } & L_{2}(\partial \omega \times(0, T)), \\
(-Q)^{1 / 2} \mathbf{z}_{N} \stackrel{N \rightarrow \infty}{\longrightarrow}(-Q)^{1 / 2} \hat{\mathbf{z}} & \text { in } & L_{2}(\partial \omega \times(0, T)) .
\end{array}
$$

$\mathbf{z}, \tilde{\mathbf{z}}, \hat{\mathbf{z}}$ satisfy bounds from (5.4).

For $\boldsymbol{\psi} \in H^{1}\left(\omega \times(0, T) \rightarrow \mathbb{R}^{n}\right), \boldsymbol{\psi}(\cdot, T)=0$, from (5.2), (5.5)-(5.7), (5.1) and the density of $H_{N}$ as $N \rightarrow \infty$,

$$
0=-\int_{\omega} \boldsymbol{\psi}(\cdot, 0) \cdot \mathbf{z}(\cdot, 0)-\int_{0}^{T} \int_{\omega} \mathbf{z} \cdot\left(\boldsymbol{\psi}_{t}+\sum_{i=1}^{m}\left(A_{i} \boldsymbol{\psi}\right)_{x_{i}}\right)-\int_{0}^{T} \int_{\omega} \mathbf{g} \cdot \boldsymbol{\psi}+\int_{0}^{T} \int_{\partial \omega} \boldsymbol{\psi} \cdot(Q \mathbf{b}+P \tilde{\mathbf{z}})
$$

Choosing $\left.\boldsymbol{\psi}\right|_{\partial \omega}=\mathbf{0}$, it follows from (5.8) that $\mathbf{z}$ satisfies (4.10) and the initial data (weakly) and that $\left.(P+Q) \mathbf{z}\right|_{\partial \omega}$ is implicitly determined. Dropping the assumption that $\boldsymbol{\psi}$ vanishes on $\partial \omega$, partial integration of (5.8) using (4.2) and (4.10) gives

$$
0=\int_{0}^{T} \int_{\partial \omega}-\mathbf{z} \cdot(P+Q) \boldsymbol{\psi}+\boldsymbol{\psi}(Q \mathbf{b}+P \tilde{\mathbf{z}})=\int_{0}^{T} \int_{\partial \omega} \boldsymbol{\psi} \cdot(Q \mathbf{b}-Q \mathbf{z})+\int_{0}^{T} \int_{\partial \omega} \boldsymbol{\psi} \cdot P(\tilde{\mathbf{z}}-\mathbf{z})
$$

for arbitrary (smooth) $\left.\psi\right|_{\partial \omega}$.

From the condition (4.4) on $P, Q$, it follows that the right-hand terms in (5.9) must vanish separately. Thus (4.11) holds. In addition, $\mathbf{z}=\tilde{\mathbf{z}}$, so there is no boundary layer. 
6. Proof of Lemma 4.3. Using $\boldsymbol{\nu}(\mathbf{x}) \cdot \mathbf{A}$ nonsingular, we have rank $P=k, \quad \operatorname{rank} Q=n-k$

for some $k$. Using (4.4) and $P, Q$ symmetric, an orthogonal basis making $P$ block diagonal will also make $Q$ block diagonal, in the form

$$
P=\left(\begin{array}{ccc}
P_{1} & \vdots & 0 \\
\cdots & \cdots & \cdots \\
0 & \vdots & 0
\end{array}\right), \quad Q=\left(\begin{array}{ccc}
0 & \vdots & 0 \\
\cdots & \cdots & \cdots \\
0 & \vdots & Q_{1}
\end{array}\right)
$$

with

$$
P_{1} \in M_{k \times k}, \quad Q_{1} \in M_{(n-k) \times(n-k)}
$$

nonsingular and symmetric.

We use the same block structure for the symmetric matrix $\left(\boldsymbol{\nu}\left(\mathbf{x}^{\prime}\right)-\boldsymbol{\nu}(\mathbf{x})\right) \cdot \mathbf{A}$,

$$
\left(\boldsymbol{\nu}\left(\mathbf{x}^{\prime}\right)-\boldsymbol{\nu}(\mathbf{x})\right) \cdot \mathbf{A}=\left(\begin{array}{ccc}
\Delta_{+} & \vdots & \Delta_{0} \\
\cdots & \cdots & \cdots \\
\Delta_{0}^{\mathrm{T}} & \vdots & \Delta_{-}
\end{array}\right)
$$

where $\Delta_{+} \in M_{k \times k}, \Delta_{0} \in M_{k \times(n-k)}, \Delta_{-} \in M_{(n-k) \times(n-k)}, \Delta_{ \pm}$symmetric.

From (6.4)

$$
\left\|\Delta_{+}\right\|,\left\|\Delta_{-}\right\|,\left\|\Delta_{-}\right\| \leq c\left|\boldsymbol{\nu}\left(\mathbf{x}^{\prime}\right)-\boldsymbol{\nu}(\mathbf{x})\right| \leq c\left|\mathbf{x}^{\prime}-\mathbf{x}\right| .
$$

We seek $P^{\prime}, Q^{\prime}$ of the form

$$
P^{\prime}=\left(\begin{array}{ccc}
P_{1}+\Delta_{+}+E & \vdots & \frac{1}{2} \Delta_{0} \\
\cdots & \ldots & \cdots \\
\frac{1}{2} \Delta_{0}^{\mathrm{T}} & \vdots & F
\end{array}\right), \quad Q^{\prime}=\left(\begin{array}{ccc}
-E & \vdots & \frac{1}{2} \Delta_{0} \\
\cdots & \cdots & \cdots \\
\frac{1}{2} \Delta_{0}^{\mathrm{T}} & \vdots & Q_{1}+\Delta_{-}-F
\end{array}\right)
$$

From (6.2), (6.4), and (6.6), clearly (4.2) holds for $P^{\prime}, Q^{\prime}$.

We find $E \in M_{k \times k}, F \in M_{(n-k) \times(n-k)}$ both symmetric so that

$$
\operatorname{rank} P^{\prime}=k
$$

and

$$
\operatorname{rank} Q^{\prime}=n-k .
$$

Using (6.6) and (6.1), since $E, F, \Delta_{ \pm}, \Delta_{0}$ are all small, (6.7) is equivalent to

$$
F=\frac{1}{4} \Delta_{0}^{\mathrm{T}}\left(P_{1}+\Delta_{+}+E\right)^{-1} \Delta_{0},
$$

and (6.8) is equivalent to

$$
E=\frac{1}{4} \Delta_{0}\left(-Q_{1}-\Delta_{-}+F\right)^{-1} \Delta_{0}^{\mathrm{T}}
$$

(Here "equivalent to" means simply that the indicated inverses exist.)

From the ordinary implicit function theorem, for $\left|\boldsymbol{\nu}\left(\mathbf{x}^{\prime}\right)-\boldsymbol{\nu}(\mathbf{x})\right|$ sufficiently small, there exists a unique $E, F$ satisfying (6.9), (6.10) simultaneously with

$$
\|E\|,\|F\| \leq c\left|\boldsymbol{\nu}\left(\mathbf{x}^{\prime}\right)-\boldsymbol{\nu}(\mathbf{x})\right| \leq c\left|\mathbf{x}^{\prime}-\mathbf{x}\right| .
$$


Equation (4.15) now follows from (6.5) and (6.11), using (6.6) and (6.2). Equation (4.4) for $P^{\prime}, Q^{\prime}$ follows from (6.7), (6.8) using (6.6), (6.2).

To prove (4.3) for $P^{\prime}, Q^{\prime}$, we observe that from (6.3), (6.5), (6.11), for $\left|\boldsymbol{\nu}\left(\mathbf{x}^{\prime}\right)-\boldsymbol{\nu}(\mathbf{x})\right|$ sufficiently small, the symmetric matrices $\left(P_{1}+\Delta_{+}+E\right),\left(-Q_{1}-\Delta_{-}+F\right)$ are strictly positive definite, while from $(6.9)$ and $(6.10), E, F$ are nonnegative definite. From (6.6), using (6.9), (6.10), for any $\mathbf{a} \in \mathbb{R}^{k}, \mathbf{b} \in \mathbb{R}^{n-k}$ not both zero,

$$
\begin{aligned}
\left(\mathbf{a}^{\mathrm{T}}, \mathbf{b}^{\mathrm{T}}\right) P^{\prime}\left(\begin{array}{l}
\mathbf{a} \\
\mathbf{b}
\end{array}\right)= & \mathbf{a}^{\mathrm{T}}\left(P_{1}+\Delta_{+}+E\right) \mathbf{a}+\mathbf{a}^{\mathrm{T}} \Delta_{0} \mathbf{b} \\
+ & \frac{1}{4}\left(\Delta_{0} \mathbf{b}\right)^{\mathrm{T}}\left(P_{1}+\Delta_{+}+E\right)^{-1} \Delta_{0} \mathbf{b} \\
= & \left|\left(P_{1}+\Delta_{+}+E\right)^{1 / 2} \mathbf{a}+\frac{1}{2}\left(P_{1}+\Delta_{+}+E\right)^{-1 / 2} \Delta_{0} \mathbf{b}\right|^{2} \\
& >0
\end{aligned}
$$

and

$$
\begin{aligned}
& \left(\mathbf{a}^{\mathrm{T}}, \mathbf{b}^{\mathrm{T}}\right) Q^{\prime}\left(\begin{array}{l}
\mathbf{a} \\
\mathbf{b}
\end{array}\right) \\
= & -\frac{1}{4}\left(\Delta^{\mathrm{T}} \mathbf{a}\right)^{\mathrm{T}}\left(-Q_{1}-\Delta_{1}+F\right)^{-1} \Delta_{0}^{\mathrm{T}} \mathbf{a}+\mathbf{a}^{\mathrm{T}} \Delta_{0} \mathbf{b} \\
+ & \mathbf{b}^{\mathrm{T}}\left(Q_{1}+\Delta_{-}-F\right) \mathbf{b} \\
= & -\left|\frac{1}{2}\left(-Q_{1}-\Delta_{1}+F\right)^{-1 / 2} \Delta_{0}^{\mathrm{T}}+\mathbf{a}\left(-Q_{1}-\Delta_{-}+F\right)^{1 / 2} \mathbf{b}\right|^{2} \\
& <0,
\end{aligned}
$$

thus proving (4.3).

Appendix A. Let

$$
\begin{aligned}
\mathbf{E}_{t} & =\nabla \times \mathbf{H} \\
\mathbf{H}_{t} & =-\nabla \times \mathbf{E}
\end{aligned}
$$

be Maxwell's equations. Here $\mathbf{E}=\left(E_{1}, E_{2}, E_{3}\right)^{T}$ and $\mathbf{H}=\left(H_{1}, H_{2}, H_{3}\right)^{T}$, we take $\Omega=\mathbb{R}^{3}$, and $\mathbf{x}_{0}=\left(x_{0}, y_{0}, z_{0}\right)=(0,0,0)$.

As a model of an antenna, we shall use an elementary or Hertz dipole. The elementary dipole is built from two time-dependent charges, $q(t)$ and $-q(t)$. The first one is located at $(0,0, d l / 2)$ and the second one at $(0,0,-d l / 2)$ in some cartesian coordinate system $\left(x^{\prime}, y^{\prime}, z^{\prime}\right)$. The electromagnetic field generated by this dipole is:

$$
\begin{aligned}
\mathbf{E}= & \hat{\mathbf{r}}\left(\frac{\dot{P}\left(t-r^{\prime}\right)}{r^{\prime 2}}+\frac{P\left(t-r^{\prime}\right)}{r^{\prime}}\right) 2 \cos \theta^{\prime} \\
& +\hat{\boldsymbol{\theta}}\left(\frac{\ddot{P}\left(t-r^{\prime}\right)}{r^{\prime}}+\frac{\dot{P}\left(t-r^{\prime}\right)}{r^{\prime 2}}+\frac{P\left(t-r^{\prime}\right)}{r^{\prime 3}}\right) \sin \theta^{\prime} \\
\mathbf{H}= & \hat{\boldsymbol{\Phi}}\left(\frac{\ddot{P}\left(t-r^{\prime}\right)}{r^{\prime}}+\frac{\dot{P}\left(t-r^{\prime}\right)}{r^{\prime 2}}\right) \sin \theta^{\prime},
\end{aligned}
$$


where

$$
\begin{aligned}
& x^{\prime}=r^{\prime} \sin \theta^{\prime} \cos \phi^{\prime}, \\
& y^{\prime}=r^{\prime} \sin \theta^{\prime} \sin \phi^{\prime}, \\
& z^{\prime}=r^{\prime} \cos \theta^{\prime} .
\end{aligned}
$$

$r^{\prime}$ and $\theta^{\prime}$ are the spherical coordinate systems, corresponding to the cartesian coordinate system $\left(x^{\prime}, y^{\prime}, z^{\prime}\right)$, and $P(t)=q(t) d l$ is the dipole moment.

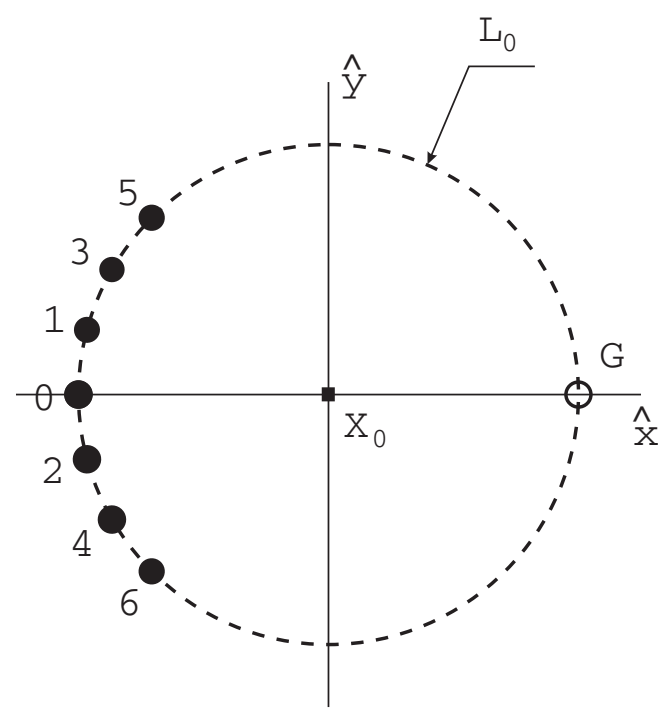

Fig. 3. A top view on the $x, y$ plane. The 'antennas', in black dots, are placed on a circle centered at $\mathbf{x}_{\theta_{j}}$ (marked by a black square) with radius $L_{0}$. The ghost antenna is placed at $\left(L_{0}, 0,0\right)$, an empty circle.

An array of 'antennas' are placed at the points $\mathbf{x}_{\theta_{j}}=\left(-\cos \theta_{j}, \sin \theta_{j}, 0\right) L_{0}$, where $\theta_{0}=0, \theta_{1}=\pi / 12, \theta_{2}=-\pi / 12, \theta_{3}=\pi / 6, \theta_{4}=-\pi / 6, \theta_{5}=\pi / 4, \theta_{6}=-\pi / 4$, and $\mathrm{a}$ 'ghost antenna' is placed at $\left(L_{0}, 0,0\right)$. The 'observer' is placed at $\mathbf{x}_{0}$; see Fig 1 .

Each 'antenna' generates an electromagnetic field (A.2), where the coordinate system $\left(x^{\prime}, y^{\prime}, z^{\prime}\right)$ for each 'antenna' is parallel to $(x, y, z)$ and is centered at $\mathbf{x}_{\theta_{j}}$. We denote these fields by $\left(\mathbf{E}\left(\theta_{j}\right), \mathbf{H}\left(\theta_{j}\right)\right)$.

If we take the following linear combination:

$$
\mathbf{u}=\left[\sum_{j=0}^{6} a_{j}\left(\begin{array}{c}
\mathbf{E}\left(\theta_{j}\right) \\
\mathbf{H}\left(\theta_{j}\right)
\end{array}\right)\right]
$$


where

$$
\begin{aligned}
a_{0} & =\frac{(-1+\sqrt{2})(-5+3 \sqrt{3})}{25-53 \sqrt{2}-15 \sqrt{3}+31 \sqrt{6}}, \\
a_{1}=a_{2} & =\frac{4-9 \sqrt{2}-2 \sqrt{3}+5 \sqrt{6}}{-25+53 \sqrt{2}+15 \sqrt{3}-31 \sqrt{6}}, \\
a_{3}=a_{4} & =\frac{14-33 \sqrt{2}-8 \sqrt{3}+19 \sqrt{6}}{25-53 \sqrt{2}-15 \sqrt{3}+31 \sqrt{6}}, \\
a_{5}=a_{6}= & 0,
\end{aligned}
$$

then the field $\mathbf{u}\left(\mathbf{x}_{0}, t\right)$ and its first derivatives are the same as the field generated by the single 'ghost antenna' placed at $\left(L_{0}, 0,0\right)$ (equivalent to substituting $\theta_{j}=\pi$ in eq. (A.2)).

Similarly, by taking

$$
\begin{array}{r}
a_{0}=-\frac{(1+\sqrt{2})(-5+3 \sqrt{3})}{25-53 \sqrt{2}-15 \sqrt{3}+31 \sqrt{6}}, \\
a_{1}=a_{2}=\frac{-2(-5-2 \sqrt{2}+3 \sqrt{3}+\sqrt{6})}{25-53 \sqrt{2}-15 \sqrt{3}+31 \sqrt{6}}, \\
a_{3}=a_{4}=\frac{38-52 \sqrt{2}-22 \sqrt{3}+30 \sqrt{6}}{25-53 \sqrt{2}-15 \sqrt{3}+31 \sqrt{6}}, \\
a_{5}=a_{6}=76+54 \sqrt{2}+44 \sqrt{3}+31 \sqrt{6},
\end{array}
$$

all of the second derivatives will coincide also.

Note that at $\mathbf{x}_{0}$, the wave coming from each of the antennae placed on $\mathbf{x}_{\theta_{j}}$ is rightmoving in the sense defined in section 1 , while the wave coming from the ghost antenna is left-moving. This result holds for any smooth function $P(t)$.

Indeed, this procedure can be continued, as discussed at the end of section 3 . Using a sufficiently large array of 'antennas', any number of derivatives can be made to coincide.

\section{REFERENCES}

[1] S. V. Tsynkov, Numerical solution of problems on unbounded domains. A review. Absorbing boundary conditions, Appl. Numer. Math. 27 (1998), no. 4, 465-532. MR1644674 (99i:65116)

[2] Advances in computational electrodynamics. The finite-difference time-domain method. Edited by Allen Taflove. Artech House Antenna Library. Artech House, Inc., Boston, MA, 1998 MR1639352 (99c:78001)

[3] J.-P. Berenger, A perfectly matched layer for the absorption of electromagnetic waves, J. Comput. Phys. 114 (1994) 185-200. MR1294924 (95e:78002)

[4] S. Abarbanel, D. Gottlieb, and J. S. Hesthaven, Long time behavior of the perfectly matched layer equations in computational electromagnetics, Proceedings of the Fifth International Conference on Spectral and High Order Methods (ICOSAHOM-01) (Uppsala). J. Sci. Comput. 17 (2002), no. 1-4, 405-422 MR1910579

[5] K. O. Friedrichs, Symmetric hyperbolic linear differential equations, CPAM 7 (1954), pp 345-392. MR0062932 (16:44c)

[6] K. O. Friedrichs, Symmetric positive linear differential equations, CPAM 11 (1958), pp. 333-418. MR0100718 (20:7147)

[7] B. Gustafsson, H.-O. Kreiss, and J Oliger. Time Dependent Problems and Difference Methods. Pure and Applied Mathematics. John Wiley \& Sons Inc., New York 1995. A Wiley-Interscience Publication. MR1377057 (97c:65145) 
[8] H.-O. Kreiss and J. Lorenz. Initial-Boundary Value Problems and the Navier-Stokes Equations, Volume 136 of Pure and Applied Mathematics. Academic Press Inc., Boston, MA, 1989. MR998379 (91a:35138) 